\title{
Eigensensitivity analysis of subgrid-scale stresses in large-eddy simulation of a turbulent axisymmetric jet
}

\author{
Lluís Jofre $^{\mathrm{a}}$, Stefan P. Domino ${ }^{\mathrm{b}}$, Gianluca Iaccarino ${ }^{\mathrm{a}}$ \\ ${ }^{a}$ Center for Turbulence Research, Stanford University, Stanford, CA 94305, USA \\ ${ }^{b}$ Computational Thermal and Fluid Mechanics, Sandia National Laboratories, \\ Albuquerque, NM 87185, USA
}

\begin{abstract}
The study of complex turbulent flows by means of large-eddy simulation approaches has become increasingly popular in many scientific and engineering applications. The underlying filtering operation of the approach enables to significantly reduce the spatial and temporal resolution requirements by means of representing only large-scale motions. However, the small-scale stresses and their effects on the resolved flow field are not negligible, and therefore require additional modeling. As a consequence, the assumptions made in the closure formulations become potential sources of model-form uncertainty that can impact the quantities of interest. The objective of this work, thus, is to perform a model-form sensitivity analysis in large-eddy simulations of an axisymmetric turbulent jet following an eigenspace-based strategy recently proposed. The approach relies on introducing perturbations to the decomposed subgrid-scale stress tensor within a range of physically plausible values. These correspond to discrepancy in magnitude (trace), anisotropy (eigenvalues) and orientation (eigenvectors) of the normalized, small-scale stresses with respect to a given tensor state, such that propagation of their effects can be assessed. The generality of the framework with respect to the six degrees of freedom of the small-scale stress tensor makes it
\end{abstract}

Email addresses: jofre@stanford.edu (Lluís Jofre), spdomin@sandia.gov (Stefan P. Domino), jops@stanford.edu (Gianluca Iaccarino) 
also suitable for its application within data-driven techniques for improved subgrid-scale modeling.

Keywords: Large-eddy simulation; Sensitivity analysis; Subgrid-scale modeling; Turbulent axisymmetric jet; Uncertainty quantification

\section{Introduction}

Large-eddy simulation (LES) has become a high-fidelity (HF) reference approach for the study of a broad range of complex turbulent flows. Some examples include wall-bounded turbulence [1], multiphase flows [2, 3], geophysical fluid dynamics $[4,5]$, and turbulent combustion [6, 7]. Compared with direct numerical simulation (DNS), LES reduces the computational cost of solving turbulent flows by applying a low-pass filter to the conservation equations. For example, the number of grid points $N$ required in LES of free shear layers scales with the Reynolds number as $N^{3} \sim R e$ [8], while resolving all the turbulent flow motions entails performing DNS of the order $N^{3} \sim R e^{9 / 4}$. The reduction in computational cost, however, is obtained at expenses of modeling the effects of the small scales on the resolved flow field in terms of subfilter stresses. Consequently, the assumptions introduced in the closure formulations become potential sources of model-form uncertainty that can affect the quantities of interest (QoI). The most common approach for closing the LES transport equations is the Boussinesq's eddy-viscosity hypothesis [9]. This type of closures represents the effects of subfilter motions on the resolved scales in analogy with the kinetic theory of gases by setting the momentum fluxes to be linearly dependent upon the rate of strain of the large scales. The imposed alignment between subfilter stresses and strain rate tends to qualitatively predict sufficient mean small-scale dissipation in homogeneous isotropic turbulence (HIT), but is typically unable to correctly mimic subfilter dynamics in more complex turbulent flows [10]. Other approaches for subfilter stress modeling are available in the literature [11]. For instance, closures based on structural assumptions $[12,13]$. One of the most 
recognized turbulent closure in this group is the similarity model [13], which is based on the assumption of scale invariance, and therefore postulates that the subfilter velocity distribution resembles the large-scale flow field. This type of models tends to be less dissipative in comparison to eddy-viscosity approaches, frequently resulting in lower stability and in the appearance of unphysical behaviors. This drawback has led to the development of mixed models [14], in which an eddy-viscosity term is added to increase subfilter dissipation.

Numerous studies have been dedicated to identify sources of error resulting from the numerical approximations required to discretely solve the LES conservation equations. Some of the most notable works are the seminal paper by Ghosal [15] and the detailed error database gathered by Meyers et al. [16]. However, even with the widespread utilization of LES in many scientific and technological fields, few studies have analyzed model-form incertitude from an uncertainty quantification (UQ) point of view. In general, most analyses are based on nonintrusive methodologies applied to simple flow configurations and are concerned mainly with sensitivities to LES closure parameters, such as model coefficients [17], filter characteristics [18] or mesh resolution [19]. A more sophisticated approach is to consider the closure parameters uncertain and estimate their effects on the QoIs by forwardpropagating them as probability distributions. This strategy has been applied to Reynolds-averaged Navier-Stokes (RANS) [20] and LES [21] models and extended to incorporate simulation data from DNS [22] and utilize Bayesian inference techniques $[10,23,24]$. In the case of complex flows, some methodologies predict on the basis of an ensemble of solutions obtained using different models, such as in earth sciences for weather and ocean forecasting $[25,26,27]$. Although common practice, all these approaches present important impediments to generalization owing to their dependency on the underlying structure of the models utilized. In this regard, the present work aims to analyze sensitivity to model-form uncertainty in LES of an axisym- 
metric turbulent jet following a systematic strategy recently presented in Jofre et al. [28]. The framework developed is inspired by an approach previously introduced in RANS modeling [29, 30, 31]. However, its extension to LES required revisiting the underlying assumptions, mathematical derivation and physical bounds of the methodology. In short, the approach is based on introducing perturbations to the decomposed, small-scale stress tensor within a range of physically plausible values. These perturbations correspond to discrepancy in magnitude (trace), shape (eigenvalues) and orientation (eigenvectors) of the normalized, small-scale stresses with respect to a given tensor state. The generality of the framework with respect to the six degrees of freedom of the small-scale stress tensor also makes it suitable for its application within data-driven techniques, like for example approaches recently developed to improve RANS predictions [32, 33, 34].

The axisymmetric, or round, turbulent jet is a canonical fluid flow found in many scientific and industrial problems. Jets are common in the natural world, for instance, in volcano eruptions, in motion and defense mechanisms of animals, in water and steam discharge of geysers, or in convective thermals in cloud physics, as well as in industrial applications involving mixing, heating and cooling, and propulsion, such as fuel injection in combustors, cooling of turbine blades, and propulsion of high-speed vessels. Most LES calculations of round turbulent jets are based on eddy-viscosity-type models or dissipative numerical schemes (e.g., [35, 36, 37, 38, 39]). The general observation in such studies is that first-order flow quantities in the axial and radial directions are well predicted, as these depend mostly on large-scale motions, whereas higher-order statistics, viz. velocity fluctuations and shear stresses, are typically not well represented. Therefore, this work systematically analyzes the impact of subfilter modeling assumptions on the QoIs to characterize underlying a priori and a posteriori differences of LES closure models. The paper is organized as follows. First, in Section 2, the LES conservation equations, together with a description of the eddy-viscosity closure 
group, are introduced. Section 3 summarizes the model-form uncertainty estimation methodology, highlighting the assumptions and choices made during the construction of the framework. A comprehensive description of the jet's reference dataset generated for this work is described in Section 4. Next, in Section 5, discrepancies between reference and base LES results are analyzed. The observations are subsequently related to sensitivity in subgridscale stress model-form uncertainty in Section 6. Finally, conclusions are drawn and future work is discussed in Section 7.

\section{Large-eddy simulation equations}

The transport LES equations are derived by applying a low-pass filter, $G$, to the Navier-Stokes equations. The filter decomposes any flow variable $\phi(\mathbf{x}, t)$ into large-, $\bar{\phi}$, and small-scale, $\phi^{\prime}$, contributions, i.e., $\phi=\bar{\phi}+\phi^{\prime}$. The filtered part is defined as

$$
\bar{\phi}(\mathbf{x}, t)=\int_{\Omega} G(\mathbf{x}, \boldsymbol{\xi}, \bar{\Delta}) \phi(\boldsymbol{\xi}, t) d \boldsymbol{\xi},
$$

with $\mathbf{x}$ and $\boldsymbol{\xi}$ position vectors in the domain $\Omega$, and $\bar{\Delta}$ the characteristic cut-off length scale of the filter.

Assuming that differentiation and filtering commute [40, 41], the filtered incompressible continuity and Navier-Stokes equations result in

$$
\frac{\partial \bar{u}_{i}}{\partial x_{i}}=0,
$$

$$
\frac{\partial \bar{u}_{i}}{\partial t}+\frac{\partial\left(\overline{u_{i} u_{j}}\right)}{\partial x_{j}}=-\frac{1}{\rho} \frac{\partial \bar{p}}{\partial x_{i}}+\nu \frac{\partial^{2} \bar{u}_{i}}{\partial x_{j} \partial x_{j}},
$$

where $u_{i}$ and $p$ are the velocity vector and pressure variables, and $\rho$ and $\nu$ are the density and kinematic viscosity of the fluid. This system is undetermined since it contains more unknowns $\left(\bar{u}_{i}, \overline{u_{i} u_{j}}, \bar{p}\right)$ than equations. Thus, in order to advance the solution of the filtered quantities in time, a closure definition 
for the nonlinear filtered advection term, $\overline{u_{i} u_{j}}$, needs to be provided, as well as boundary conditions and an initial state for $\bar{u}_{i}$ and $\bar{p}$.

In a LES framework, Leonard's decomposition [42] separates $\overline{u_{i} u_{j}}$ into a large-scale part, $\bar{u}_{i} \bar{u}_{j}$, and a subfilter scale (SFS), or turbulent, stress tensor part, $\tau_{i j}=\bar{u}_{i} u_{j}-\bar{u}_{i} \bar{u}_{j}$. As a result, the conservation of filtered momentum can be recast in the form

$$
\frac{\partial \bar{u}_{i}}{\partial t}+\frac{\partial\left(\bar{u}_{i} \bar{u}_{j}\right)}{\partial x_{j}}=-\frac{1}{\rho} \frac{\partial \bar{p}}{\partial x_{i}}+\nu \frac{\partial^{2} \bar{u}_{i}}{\partial x_{j} \partial x_{j}}-\frac{\partial \tau_{i j}}{\partial x_{j}} .
$$

The resolved scales of LES, $\bar{\phi}$, are characterized by the filter applied to the conservation equations. In a general context, the filtering and discretization operators are different [43]. However, in most cases the spatial discretization is chosen to be specifically the low-pass filter [44], and therefore $\tau_{i j}$ is habitually referred to as the subgrid-scale (SGS) tensor.

\subsection{Subgrid-scale models}

The objective of SGS models is to replace the unknown value of $\tau_{i j}$ by an approximate representation. In order to clearly differentiate $\tau_{i j}$ from its approximation, $\tau_{i j}^{S G S}$ will be used in this paper to refer to the modeled $\tau_{i j}$. Many different models for $\tau_{i j}^{S G S}$ exist, e.g., [12, 13, 14, 11]. However, the eddy-viscosity assumption [44] is the most popular closure due to its robustness and ease of implementation. This group of models represents the deviatoric part of $\tau_{i j}$ as

$$
\tau_{i j}^{S G S}-\frac{\tau_{k k}^{S G S}}{3} \delta_{i j}=-2 \nu_{S G S} \bar{S}_{i j},
$$

where $\tau_{k k}$ is the trace of the tensor, $\delta_{i j}$ is the Kronecker delta, $\nu_{S G S}$ is the turbulent viscosity given by a specific model, e.g., [4, 45, 46, 47, 48], and $\bar{S}_{i j}=1 / 2\left(\partial \bar{u}_{i} / \partial x_{j}+\partial \bar{u}_{j} / \partial x_{i}\right)$ is the rate-of-strain tensor of the resolved scales. As it can be observed in the equation above, the different eddyviscosity models only differ in the evaluation of $\nu_{S G S}$. Therefore, they only 
account for variability in the magnitude of the tensor, while the anisotropy and orientation are directly determined by $\bar{S}_{i j}$. In other words, this group of models focuses only in one of the six degrees of freedom in $\tau_{i j}$. For example, the Wall-Adapting Local Eddy-Viscosity (WALE) model [46] evaluates the turbulent viscosity dynamically as

$\nu_{S G S}=\left(C_{w} \Delta\right)^{2} \frac{\left(\mathcal{S}_{i j}^{d} \mathcal{S}_{i j}^{d}\right)^{3 / 2}}{\left(\bar{S}_{i j} \bar{S}_{i j}\right)^{5 / 2}+\left(\mathcal{S}_{i j}^{d} \mathcal{S}_{i j}^{d}\right)^{5 / 4}} \quad$ and $\quad \mathcal{S}_{i j}^{d}=\frac{1}{2}\left(\bar{g}_{i j}^{2}+\bar{g}_{j i}^{2}\right)-\frac{\bar{g}_{k k}^{2}}{3} \delta_{i j}$

with $C_{w}$ a model coefficient $\left(C_{w}=0.325\right.$ based on homogeneous isotropic turbulence data), $\Delta$ the subgrid characteristic length scale (size of the mesh in implicit filtering), and $\bar{g}_{i j}=\partial \bar{u}_{i} / \partial x_{j}$ the velocity gradient tensor of the resolved scales.

An additional parameter requiring modeling is the trace of the tensor, $\tau_{k k}^{S G S}$, as it has been subtracted from $\tau_{i j}^{S G S}$ in Eq. 5. However, in LES of incompressible flows, the isotropic part, $\tau_{k k}^{S G S} / 3$, is usually added to the filtered pressure, resulting in a modified pressure that the LES solver evolves in time. In the case of compressible flows, explicit subgrid-scale models have been proposed for $\tau_{k k}^{S G S}$, like for example the parametrization by Yoshizawa [49]

$$
\tau_{k k}^{S G S}=2 C_{I} \Delta^{2}\left|\bar{S}_{i j}\right|^{2} \quad \text { with } \quad\left|\bar{S}_{i j}\right|=\left(2 \bar{S}_{i j} \bar{S}_{i j}\right)^{1 / 2},
$$

where $C_{I}$ is a model coefficient that can be approximated, for instance, as proposed by Moin et al. [50], or a different approach by Vreman et al. [51] which models $\tau_{k k}^{S G S}$ as

$$
\tau_{k k}^{S G S}=4 C_{w} \Delta^{2} \sum_{i, j} \bar{S}_{i j}^{2}
$$

In this work, we follow the second approach, Eq. 8, to model $\tau_{k k}$. 
that guarantee the spectrum of $\overline{u_{i} u_{j}}$ to be non-negative and real.

\footnotetext{
${ }^{1}$ The summation convention is adopted for Latin, but not for Greek indices.
} 


\subsection{Tensor decomposition}

The nonlinear filtered advection term can be decomposed into factors by introducing the normalized anisotropy tensor, $\bar{a}_{i j}$, as

$$
\bar{a}_{i j}=\frac{\overline{u_{i} u_{j}}}{\overline{u_{k} u_{k}}}-\frac{1}{3} \delta_{i j}=\bar{v}_{i n} \bar{\Lambda}_{n l} \bar{v}_{j l}
$$

which is symmetric and trace-free, i.e., the eigenvalues sum zero. Its eigendecomposition is given by a matrix of orthonormal eigenvectors, $\bar{v}_{i n}$, and a diagonal matrix of eigenvalues, $\bar{\Lambda}_{n l}$, ordered such that $\bar{\lambda}_{1} \geq \bar{\lambda}_{2} \geq \bar{\lambda}_{3}$. As a result, the anisotropy tensor allows reformulating $\overline{u_{i} u_{j}}$ in terms of magnitude, $\overline{u_{k} u_{k}}$, shape, $\bar{\Lambda}_{n l}$, and orientation, $\bar{v}_{i n}$, in the form

$$
\overline{u_{i} u_{j}}=\overline{u_{k} u_{k}}\left(\bar{v}_{i n} \bar{\Lambda}_{n l} \bar{v}_{j l}+\frac{1}{3} \delta_{i j}\right) .
$$

\subsection{Barycentric map}

Three limiting states exist in the case of a positive semi-definite secondorder tensor: (i) one-component (rod-like) where $2 / 3=\lambda_{1}>\lambda_{2}=\lambda_{3}=$ $-1 / 3$, (ii) two-component axisymmetric (disk-like) with $1 / 6=\lambda_{1}=\lambda_{2}>$ $\lambda_{3}=-1 / 3$, and (iii) three-component (isotropic) characterized by $\lambda_{1}=$ $\lambda_{2}=\lambda_{3}=0$. The anisotropy tensor shapes and their limiting states can be visualized, for instance, in terms of the barycentric map [53], which relies on the fact that any anisotropy state is a convex combination of the limiting states of componentiality. In an Euclidean space, these can be represented as the vertices of an equilateral triangle with coordinates $\mathbf{x}_{1 c}=(0,0), \mathbf{x}_{2 c}=$ $(1,0)$, and $\mathbf{x}_{3 c}=(1 / 2, \sqrt{3} / 2)$. A graphical representation of the map and the different anisotropy shapes is illustrated in Figure 1. One of the main advantages is that it provides a linear relation between anisotropy eigenvalues and Euclidean space through the projection

$$
\mathbf{x}=\mathbf{x}_{1 c}\left(\lambda_{1}-\lambda_{2}\right)+2 \mathbf{x}_{2 c}\left(\lambda_{2}-\lambda_{3}\right)+\mathbf{x}_{3 c}\left(3 \lambda_{3}+1\right),
$$



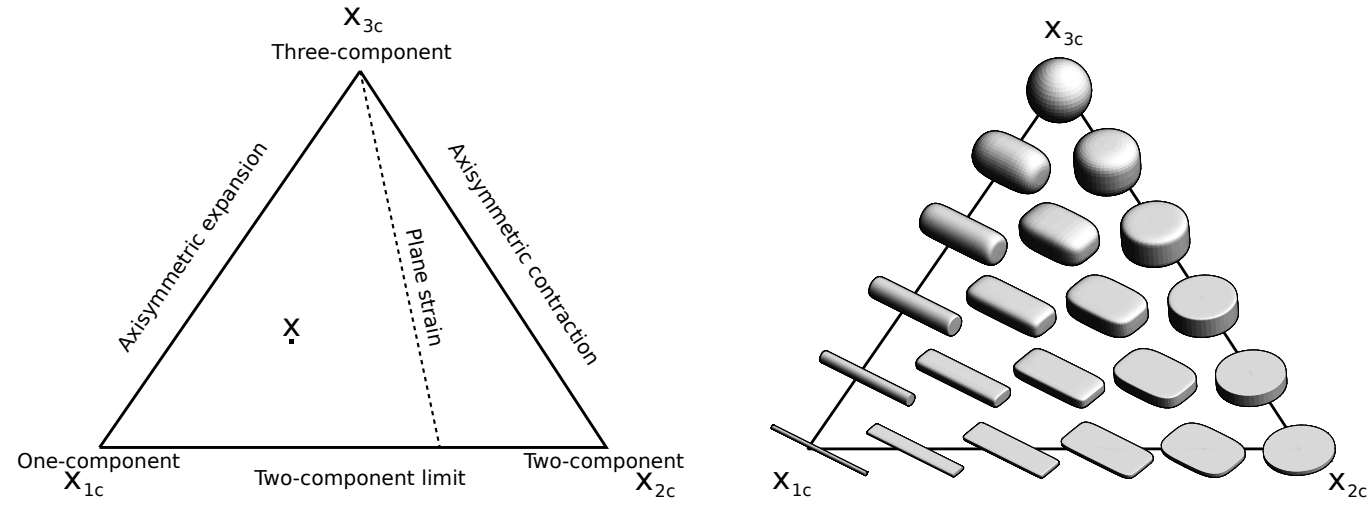

Figure 1: Barycentric map based on the eigenvalues of a general second-order anisotropy tensor. (left) Limiting states of componentiality. (right) Tensor shapes visualized with superquadric glyphs [54] (figure regenerated using open-source software [55]).

where $a_{i j}^{\text {res }}$ and $a_{i j}^{S G S}$ are the resolved and SGS components of the total anisotropy tensor given by

$$
a_{i j}^{r e s}=\frac{1}{\overline{u_{k} u_{k}}}\left(\bar{u}_{i} \bar{u}_{j}-\frac{\bar{u}_{k} \bar{u}_{k}}{3} \delta_{i j}\right) \quad \text { and } \quad a_{i j}^{S G S}=\frac{1}{\overline{u_{k} u_{k}}}\left(\tau_{i j}^{S G S}-\frac{\tau_{k k}^{S G S}}{3} \delta_{i j}\right),
$$


with $\bar{u}_{k} \bar{u}_{k}$ the resolved part of $\overline{u_{k} u_{k}}$. Once the separation between resolved and modeled parts is performed, perturbations are defined as

$$
{\overline{u_{i} u_{j}}}^{*}=\bar{u}_{i} \bar{u}_{j}+\tau_{i j}^{S G S^{*}}=\bar{u}_{i} \bar{u}_{j}+{\overline{u_{k} u_{k}}}^{*} a_{i j}^{S G S^{*}}+\frac{\tau_{k k}^{S G S^{*}}}{3} \delta_{i j},
$$

with ${\overline{u_{k} u_{k}}}^{*}=\bar{u}_{k} \bar{u}_{k}+\tau_{k k}^{S G S^{*}}$ and $a_{i j}^{S G S^{*}}=v_{i n}^{S G S^{*}} \Lambda_{n l}^{S G S^{*}} v_{j l}^{S G S^{*}}$. Thus, perturbations (indicated with ${ }^{*}$ ) are applied to the subgrid scales only, and are specified as a discrepancy of the SGS tensor in terms of magnitude $\left(\tau_{k k}^{S G S^{*}}=\tau_{k k}^{S G S}+\Delta \tau_{k k}^{S G S}\right)$, shape (diagonal matrix $\Lambda_{n l}^{S G S^{*}}$ of perturbed eigenvalues $\left.\lambda_{l}^{*}\right)$, and orientation $\left(v_{i j}^{S G S^{*}}=q_{i n} v_{n j}^{S G S}\right.$ with $q_{i n}$ an orthonormal rotation matrix).

\subsubsection{Modeled SGS stress tensor magnitude perturbation}

Lower and upper bounds for the perturbation of $\tau_{k k}^{S G S}$ can be obtained by considering the sign nature of the quantities composing the trace of the nonlinear filtered advection term. Its mathematical expression is

$$
\overline{u_{k} u_{k}}=\bar{u}_{k} \bar{u}_{k}+\tau_{k k}^{S G S},
$$

where $\overline{u_{k} u_{k}}$ and $\bar{u}_{k} \bar{u}_{k}$ are non-negative. The former, $\overline{u_{k} u_{k}}$, is non-negative due to the restriction made in this work that realizability conditions apply to $\overline{u_{i} u_{j}}$, whereas the latter, $\bar{u}_{k} \bar{u}_{k}$, is non-negative by construction independently of the filter utilized, given its square product expression. In order to respect these properties, any possible perturbation of $\tau_{k k}^{S G S}$ is bounded by $\overline{u_{k} u_{k}}=$ $\bar{u}_{k} \bar{u}_{k}+\tau_{k k}^{S G S} \geq 0$ and $\bar{u}_{k} \bar{u}_{k}=\overline{u_{k} u_{k}}-\tau_{k k}^{S G S} \geq 0$. Therefore, the interval of magnitude discrepancy written in terms of $\Delta \tau_{k k}^{S G S}$ results in

$$
-\bar{u}_{k} \bar{u}_{k}-\tau_{k k}^{S G S} \leq \Delta \tau_{k k}^{S G S} \leq \overline{u_{k} u_{k}}-\tau_{k k}^{S G S}
$$




\subsubsection{Modeled SGS stress tensor eigenvalue perturbation}

Different strategies can be constructed to perturb the eigenvalues of $a_{i j}^{S G S}$ since the framework utilized allows the perturbations to be defined implicitly through the coordinates on the barycentric map as $\lambda_{l}^{S G S^{*}}=\mathbf{B}^{-1} \mathbf{x}^{S G S^{*}}$. For this study, we choose the uncertainty to be characterized by a direction, $\mathbf{x}^{t}-\mathbf{x}^{S G S}$, and a magnitude, $\left\|\mathbf{x}^{t}-\mathbf{x}^{S G S}\right\|$, both of which can vary in space and time. In particular, perturbations within the barycentric map are considered toward each of the three corners of the triangle, namely $\mathbf{x}_{1 c}, \mathbf{x}_{2 c}$, and $\mathbf{x}_{3 c}$, and are defined by means of a relative distance $\Delta_{B}=\left\|\mathbf{x}^{S G S^{*}}-\mathbf{x}^{S G S}\right\| /\left\|\mathbf{x}^{t}-\mathbf{x}^{S G S}\right\|$ toward the target vertex. In mathematical form, the eigenvalue perturbation can be expressed through the following translation

$$
\mathbf{x}^{S G S^{*}}=\mathbf{x}^{S G S}+\Delta_{B}\left(\mathbf{x}^{t}-\mathbf{x}^{S G S}\right)
$$

where $\mathbf{x}^{S G S}, \mathbf{x}^{S G S^{*}}$, and $\mathbf{x}^{t}$ are the coordinates of the base-model prediction, new perturbed position and target corner, respectively. Applying the linear map $\mathbf{B}$ to the new position $\mathbf{x}^{S G S^{*}}$, the perturbed eigenvalues are uniquely defined as

$$
\lambda_{l}^{S G S^{*}}=\left(1-\Delta_{B}\right) \lambda_{l}^{S G S}+\Delta_{B} \lambda_{l}^{t}
$$

\subsubsection{Modeled SGS stress tensor eigenvector perturbation}

The methodology to introduce perturbations into the eigenvectors of $a_{i j}^{S G S}$ is based on the physical constraints of energy transfer between resolved and modeled scales. The starting point is the balance equation for resolved filtered kinetic energy, $E_{f}=\bar{u}_{k} \bar{u}_{k} / 2$, given as [8]

$$
\frac{\partial E_{f}}{\partial t}+\bar{u}_{j} \frac{\partial E_{f}}{\partial x_{j}}-\frac{\partial}{\partial x_{i}}\left[\bar{u}_{j}\left(2 \nu \bar{S}_{i j}-\tau_{i j}^{r}-\frac{1}{\rho} \bar{p} \delta_{i j}\right)\right]=-\epsilon_{f}-\mathcal{P}_{r} .
$$

The terms on the left-hand side represent transport, while the terms on the right-hand side correspond to viscous dissipation, $\epsilon_{f}=2 \nu \bar{S}_{i j} \bar{S}_{i j}$, and rate of production of SGS kinetic energy, $\mathcal{P}_{r}=-\tau_{i j}^{r} \bar{S}_{i j}$, with $\tau_{i j}^{r}=\tau_{i j}$ 
$\tau_{k k} \delta_{i j} / 3$ the deviatoric part of the SGS stresses. The latter is of particular interest since it represents the transfer of kinetic energy between resolved and modeled scales. In three-dimensional (3-D), single-phase turbulence, $\mathcal{P}_{r}$ transfers energy from large to small scales in a statistically-averaged sense, i.e., forward-scatter. However, it can present positive or negative values instantaneously, and therefore it can act as a sink (forward-scatter) or source (backscatter) term for $E_{f}[56]$.

In the above equation, the transport of SGS stresses, $\partial\left(\bar{u}_{j} \tau_{i j}^{r}\right) / \partial x_{i}$, and $\mathcal{P}_{r}$ require closure through $\tau_{i j}^{r}$. However, modeling $\partial\left(\bar{u}_{j} \tau_{i j}^{r}\right) / \partial x_{i}$ is significantly complex as it involves explicit differentiation operations. By contrast, the closure of $\mathcal{P}_{r}$ is more amenable since $\tau_{i j}^{S G S}$ is typically closed based on single-point information. The value of the inner product $\mathcal{P}_{r}=-\operatorname{tr}\left(\tau_{i j}^{r} \bar{S}_{i j}\right)$ depends on the alignment between the eigenvectors of $\tau_{i j}^{r}$ and $\bar{S}_{i j}$. Diverse alignments between these two tensors can be considered. However, for the purpose of enveloping the possible dynamics, the methodology utilized seeks the extremal values of this inner product. In the case of $\tau_{i j}^{r}$ being real and $\bar{S}_{i j}$ real symmetric, the lower and upper bounds are given by the following expression [57]

$$
\lambda_{1} \gamma_{3}+\lambda_{2} \gamma_{2}+\lambda_{3} \gamma_{1} \leq \mathcal{P}_{r} \leq \lambda_{1} \gamma_{1}+\lambda_{2} \gamma_{2}+\lambda_{3} \gamma_{3},
$$

with $\lambda_{l}$ and $\gamma_{l}$ the eigenvalues of $\tau_{i j}^{r}$ and $\bar{S}_{i j}$, respectively. The upper bound in this inequality corresponds to the situation in which $\tau_{i j}^{r}$ and $\bar{S}_{i j}$ share the same basis of eigenvectors, while the lower bound is the case in which the eigenvector bases are the same except for a permutation between the first and third eigenvectors. From a practical perspective, the existence of these bounds suggests that only two eigenvector sets need to be considered. These can be easily analyzed by setting the perturbed eigenvectors of $\tau_{i j}^{S G S}$ to be the eigenvectors of $\bar{S}_{i j}$ with and without a permutation of its first and third eigenvectors. In the case of eddy-viscosity-type SGS models, the rate of production of SGS kinetic energy expressed in terms of the eigenvalues of 
$\bar{S}_{i j}$ simplifies to

$$
\mathcal{P}_{r}=2 \nu_{S G S}\left(\gamma_{1}^{2}+\gamma_{2}^{2}+\gamma_{3}^{2}\right)
$$

which is non-negative if $\nu_{S G S} \geq 0$, and therefore acts as a kinetic energy sink (forward-scatter) in Eq. 22. If the orientation of $\tau_{i j}^{S G S}$ is rotated with respect to $\bar{S}_{i j}$ by a permutation of the first and third eigenvectors, the above expression is modified to

$$
\mathcal{P}_{r}=2 \nu_{S G S}\left(\gamma_{1}^{2}+4 \gamma_{1} \gamma_{3}+\gamma_{3}^{2}\right),
$$

with $\gamma_{1} \gamma_{3} \leq 0$ since $\gamma_{1}+\gamma_{2}+\gamma_{3}=0$ (incompressible flow) and $\gamma_{1} \geq \gamma_{2} \geq \gamma_{3}$. As a result, if $\left|\gamma_{1}\right| /\left|\gamma_{3}\right| \sim 1$ and $\nu_{S G S} \geq 0, \mathcal{P}_{r}$ is non-positive, and consequently increases the turbulence intensity by energizing the large eddies from the SGS scales (backscatter).

\section{Description of the numerical reference dataset}

The flow studied in this work is based on the round jet experimentally studied by Amielh et al. [58]. The experimental data are utilized to validate the numerical dataset generated in this work. Numerical results of the flow are obtained by means of the unstructured and massively parallel low-Machnumber flow solver Nalu [59, 60, 61].

\subsection{Jet configuration and computational setup}

The flow corresponds to an axisymmetric turbulent jet at $R e=U_{j} D_{j} / \nu=$ 21000 based on the axial velocity at the jet exit, $U_{j}=12 \mathrm{~m} / \mathrm{s}$, the jet nozzle diameter, $D_{j}=2.6 \cdot 10^{-2} \mathrm{~m}$, and the kinematic viscosity of the fluid, $\nu$. As schematically illustrated in Figure 2, the jet discharges from a long pipe (modeled with periodic boundaries) into a slow coflow, $U_{e}=9 \cdot 10^{-1} \mathrm{~m} / \mathrm{s}$, of the same fluid. Subscripts $j$ and $e$ correspond to the jet flow and external coflow, respectively. The ratios between pipe diameter and computational domain are $D_{e} / D_{j}=20$ and $L / D_{j}=30$. The inlet jet velocity is extracted 

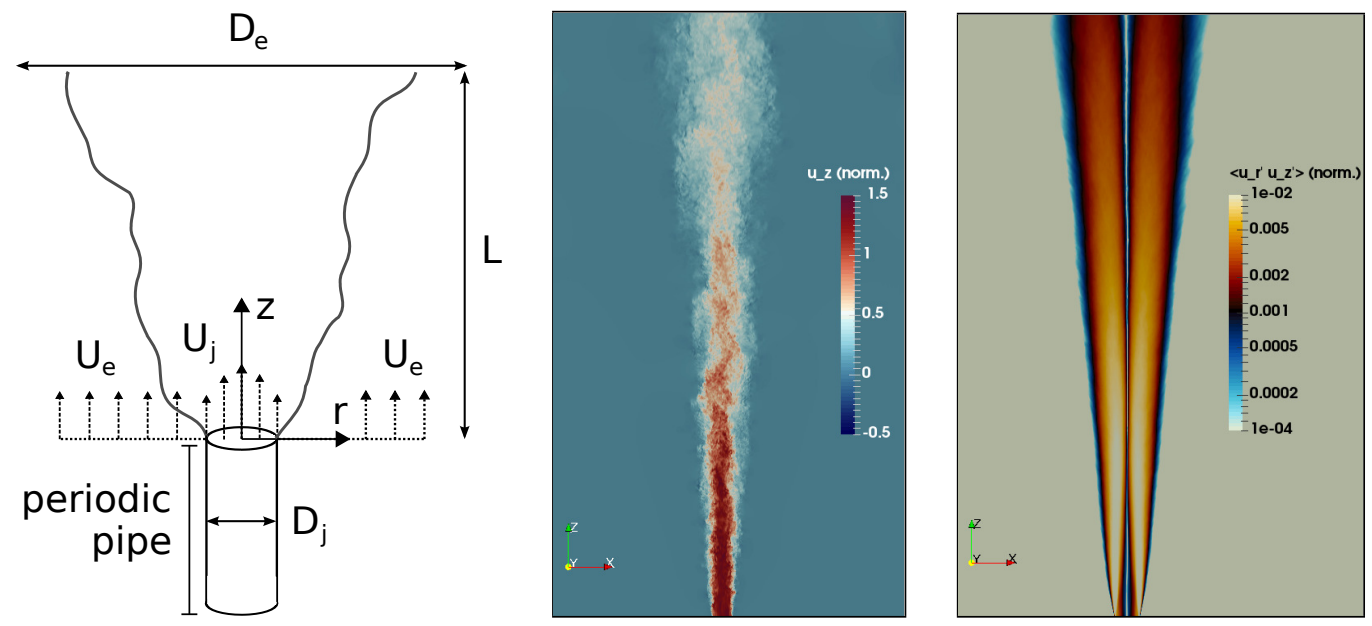

Figure 2: Axisymmetric turbulent jet at $R e=21000$. (left) Sketch of the computational setup. (center) Instantaneous snapshot of normalized axial velocity $u_{z} /\left(U_{j}-U_{e}\right)$ on the $x z$-plane. (right) Normalized Reynolds shear-stress $\left\langle u_{r}^{\prime} u_{z}^{\prime}\right\rangle /\left(U_{j}-U_{e}\right)^{2}$ on the $x z$-plane.

from a plane perpendicular to the axis of a periodic turbulent pipe flow with momentum flux $M_{j}=1 \cdot 10^{-1} \mathrm{~N}$, a uniform velocity profile is utilized for the co-flow, and specified-pressure open boundary conditions are imposed at the exit and lateral surfaces of the domain. All simulations start from the jet discharging into a fluid with initial velocity $U_{e}$. The averaging is started once a sufficiently long transient period is surpassed, $t U_{j} / L \approx 10$, and statistics are collected over a time period of $\Delta t U_{j} / D_{j} \approx 1000$. Additional averaging is performed in the azimuthal direction.

The mesh designed to carefully perform high-fidelity simulations of the flow (DNS-like resolution) is based on scaling arguments for free shear flows [62]. The timescale of the large eddies in a turbulent jet can be estimated as $t_{l} \sim D_{j} / U_{j}$. If it is assumed that the kinetic energy supply rate is proportional to the inverse of this timescale, the dissipation rate can be approximated by $\epsilon \sim U_{j}^{3} / D_{j}$. As a result, the Kolmogorov length scale can be estimated as $\eta \equiv\left(\nu^{3} / \epsilon\right)^{1 / 4} \sim D_{j} / R e^{3 / 4}$. Following this scaling, the computational domain is spatially discretized by means of an axisymmetric mesh of approximately $200 \mathrm{M}$ control volumes with resolutions of $\Delta / \eta \sim \mathcal{O}$ (1) in 

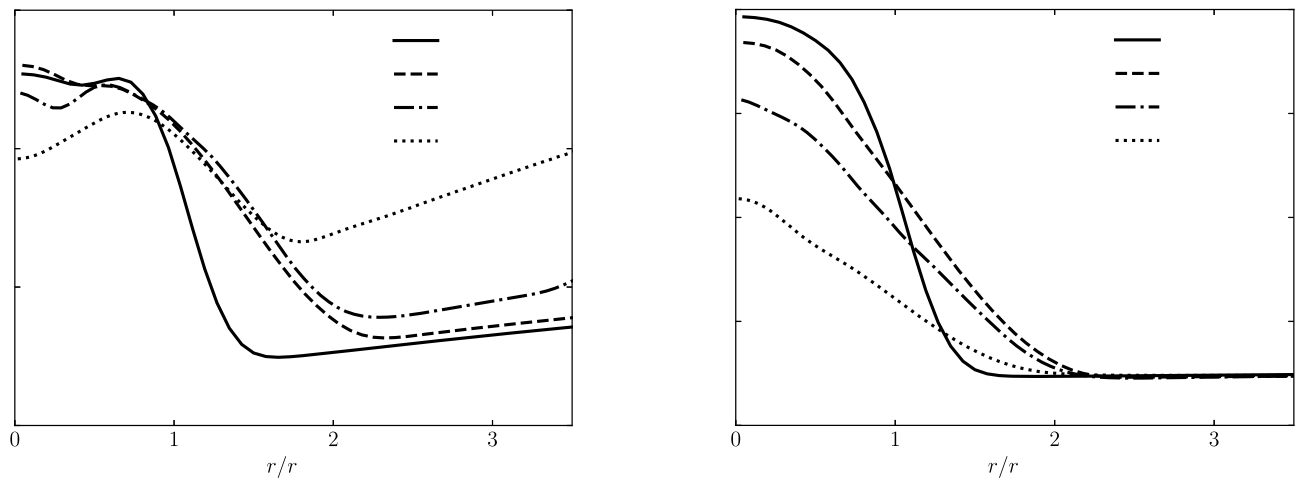

Figure 3: Radial profiles of grid size (a) and jet diameter (b) to Kolmogorov scale ratios at different axial positions.

the cylindrical region $0<r / D_{j}<5$. As shown in Figure 3, this assumption has been verified a posteriori by extracting the Kolmogorov scale, $\eta$, directly from the numerical data and comparing its value to the grid size, $\Delta$, and jet diameter, $D_{j}$, along radial profiles at axial positions $z / D_{j}=1,5,10,20$; (i) the ratio $\Delta / \eta$ is $\mathcal{O}(1)$ for all profiles and presents a rapid variation in the region $r / r_{1 / 2} \approx 1$, and (ii) the separation of flow scales $D_{j} / \eta$ is maximum at the jet axis with ratios of $\mathcal{O}\left(R e^{3 / 4}\right)$.

\subsection{Characterization of the flow}

Visualizations of an instantaneous axial velocity snapshot and Reynolds shear-stress obtained from the numerical dataset are depicted in Figure 2. The velocity difference between the jet and co-flow generates a highly unstable, thin shear layer. This shear layer continuously grows downstream as a result of Kelvin-Helmholtz flow instabilities that eventually lead to the generation of strong turbulent fluctuations. The resulting highly turbulent shear flow entrains ambient fluid into the jet and enhances the flow mixing. Consequently, the shear layer spreads in the radial direction and the jet velocity decreases. The spreading of the shear layer reduces the potential core of the jet, i.e., central region with an almost uniform velocity, which disappears when shear layers from all sides merge. The entrainment and mixing 
continues downstream the potential core region where the axial velocity distribution continuously flattens following a bell-shaped profile and becomes self-similar for $z / D_{j}>30$.

In the next section, focus will be placed on analyzing the differences between $\tau_{i j}$ calculated from filtering the numerical dataset to directly evaluate $\bar{\tau}_{i j}$, and utilizing the WALE SGS model, $\tau_{i j}^{S G S}$, at different axial and radial positions. Of particular interest is the jet's half-width radial position $r_{1 / 2}$, defined as $\left(\left\langle u_{z}\left(r_{1 / 2}, 0, z\right)\right\rangle-U_{e}\right) /\left(\left\langle u_{z}(0,0, z)\right\rangle-U_{e}\right)=1 / 2$, as it characterizes the region of the flow exhibiting maximum production of turbulent kinetic energy, $\mathcal{P} \equiv-\left\langle u_{i}^{\prime} u_{j}^{\prime}\right\rangle \partial\left\langle u_{i}\right\rangle / \partial x_{j}$; this term quantifies the transfer of kinetic energy from the mean flow to the fluctuating velocity field as a result of the interaction between the mean velocity gradients, $\partial\left\langle u_{i}\right\rangle / \partial x_{j}$, and Reynolds stresses, $R_{i j}=\left\langle u_{i}^{\prime} u_{j}^{\prime}\right\rangle$. For the flow studied in this work, $\mathcal{P}$ is positive, and therefore acts as a source in the transport equation for the turbulent kinetic energy (TKE), $k \equiv 1 / 2\left\langle u_{k}^{\prime} u_{k}^{\prime}\right\rangle$. As shown in Figure $4, \mathcal{P}$ peaks in the region defined by $r / r_{1 / 2} \approx 1$ for the two different axial positions. The peak in the radial profiles of $k$ displays a similar trend in the near-field region of the jet, viz. $z / D_{j}=5$, but it broadens to smaller $r / r_{1 / 2}$ away from the nozzle as a result of a decay in $\mathcal{P}$ combined with increased turbulence mixing. In particular, only the symmetric part of the mean velocity gradient tensor, $\left\langle S_{i j}\right\rangle$, and, in the case of incompressible flow, the anisotropic part of the Reynolds stresses, $a_{i j}=R_{i j}-2 k \delta_{i j} / 3$, affect production, i.e., $\mathcal{P}=-a_{i j}\left\langle S_{i j}\right\rangle$. The maximum value of this inner product for this type of flow is found in the region $r / r_{1 / 2} \approx 1$ as represented on the barycentric map depicted in Figure 4 for a radial profile at $z / D_{j}=1$. The location of maximum $\mathcal{P}$ corresponds to Reynolds stresses presenting axisymmetric expansion. This stress topology is indicative of a region of high strain/dissipation that is undergoing compression in one direction and extension in the other two as it is characteristic in shear layers [63]. 

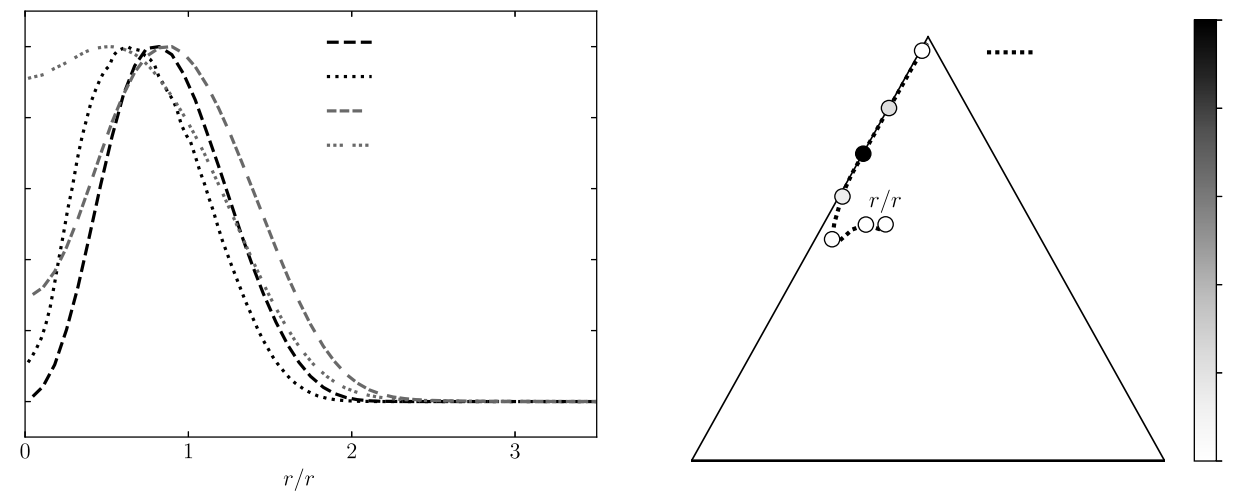

Figure 4: (a) Radial profiles of turbulent kinetic energy production and distribution at different axial positions. (b) Radial profile of Reynolds stresses at $z / D_{j}=1$ represented on the barycentric map.

\subsection{Comparison against experimental data}

Comparison of first- and second-order flow statistics between experimental and reference numerical results are shown in Figure 5. Complete agreement is observed for the mean axial velocity along the jet axis and for radial profiles at axial positions $z / D_{j}=5,10,20$. These mean quantities characterize the potential core decay and spread angle of the jet, and therefore are function of the shear layer evolution. In terms of Reynolds normal, $\left\langle u_{z}^{\prime}\right\rangle$ and $\left\langle u_{r}^{\prime}\right\rangle$, and shear, $\left\langle u_{r}^{\prime} u_{z}^{\prime}\right\rangle$, stresses, the agreement is extremely good except for the radial and shear stresses at $z / D_{j}=5,20$ where minor differences are observed for $r / r_{1 / 2} \approx 1$ that may fall within the experiment uncertainty (not documented in Amielh et al. [58]). It is important to notice the significant anisotropy exhibited by the Reynolds stresses as shown by the shear stresses and the increase in deviation of normal stresses away from the nozzle. Similar to the first-order quantities, the turbulent kinetic energy is completely well predicted by the numerical dataset at the different axial positions, which indicates very good agreement of the isotropic part of the Reynolds stresses with the experiment and provides additional confidence on the accuracy of the normal stresses obtained. These results demonstrate that the numerical 
simulation is able to accurately capture the main physical mechanisms responsible for the instability of the shear layer and the subsequent entrainment and mixing processes characteristic of free shear flows.

\section{Discrepancy between reference and LES results}

The first step of estimating model-form uncertainty is to characterize discrepancies between $\tau_{i j}$ evaluated from filtering the numerical dataset to directly calculate, $\bar{\tau}_{i j}$, and $\tau_{i j}^{S G S}$ based on the WALE SGS model. The $\bar{\tau}_{i j}=$ $\overline{u_{i} u_{j}}-\bar{u}_{i} \bar{u}_{j}$ data are obtained by filtering five instantaneous velocity field snapshots at different flow through times (FTT), defined as FTT $\sim L / U_{j}$, of the numerical dataset described in Section 4. The filtering operation is carried out by means of a second-order Gaussian filter defined as [64]

$$
\bar{\phi}=\phi+\frac{\bar{\Delta}^{2}}{24} \frac{\partial^{2} \phi}{\partial x_{i}^{2}}+\mathcal{O}\left(\bar{\Delta}^{4}\right)
$$

Data for the WALE-SGS-modeled $\tau_{i j}^{S G S}$ are computed (i) a priori from the filtered snapshots in Sections 5.2, 5.3, and 5.4, and (ii) a posteriori by performing LES on the computational setup described in Section 4 on a mesh of approximately $3 \mathrm{M}$ control volumes and with a resolution with respect to the DNS-like mesh of $\Delta_{\mathrm{LES}} / \Delta_{\mathrm{DNS}} \sim 4$ in Section 5.1. Similar to the filtered data, five instantaneous velocity field snapshots at different FTTs are utilized for the a priori analysis. The filter width in Eq. 26 is set to an equivalent LES mesh resolution of $\bar{\Delta} / \Delta_{\mathrm{LES}} \sim 1$. The ratio of averaged turbulent viscosity, $\nu_{S G S}$, obtained from the LES a posteriori calculation to kinematic viscosity, $\nu$, is depicted in Figure 6 showing that the regions of the flow in which the SGS model is more active correspond to the shear layers with values $\left\langle\nu_{S G S}\right\rangle / \nu \sim 3 \div 5$.

\subsection{Comparison of LES against the numerical reference dataset}

Prior to presenting the differences between filtered and modeled tensors from reference data, Figure 7 summarizes the accuracy of the LES compared 
with that of the DNS-like numerical dataset in terms of first- and secondorder statistics. The extension of the potential core and axisymmetric decay in the interaction region is underpredicted as shown by the mean axial velocity along the jet axis $\left(2<z / D_{j}<22\right)$ and the corresponding radial profiles (Figure $7(\mathrm{a}, \mathrm{b})$ ). This underprediction is connected to large deviatoric stresses near the jet nozzle $\left(z / D_{j} \leq 5\right)$ indicative of a rapid development of the shear layer (Figure 7(e)) as a result of flow instabilities growing too fast. Far downstream in the fully developed region $\left(z / D_{j}>25\right)$, where turbulent mixing prevails, the axial velocity recovers to match the reference data, while the shear stresses become underestimated owing to the prematurely development of the shear layer. The flow in the outer layer region, which is dominated by large-scale entrainment motions, is well predicted as shown by the radial profiles above $r / r_{1 / 2} \approx 1$ collapsing with the reference dataset. The normal stresses, and their aggregate representation through the turbulent kinetic energy, follow the same trend as the mean and shear stress statistics, viz. overprediction near the jet nozzle $\left(z / D_{j} \leq 5\right)$ and underestimation in the developed flow region $\left(z / D_{j} \geq 10\right)$ for $r / r_{1 / 2}<2$, whereas significantly good agreement with the reference results for the outer layer region is shown.

\subsection{Correlation coefficient discrepancy}

The conventional procedure to analyze discrepancy between filtered and modeled $\tau_{i j}$ is to calculate the correlation coefficient between the two tensors by means of the normalized inner product [12]

$$
C\left(\bar{\tau}_{i j}, \tau_{i j}^{S G S}\right)=\left\langle\bar{\tau}_{i j} \tau_{i j}^{S G S}\right\rangle /\left(\left\langle\bar{\tau}_{i j} \bar{\tau}_{i j}\right\rangle^{1 / 2}\left\langle\tau_{i j}^{S G S} \tau_{i j}^{S G S}\right\rangle^{1 / 2}\right),
$$

which is $C=1$ for perfectly correlated tensors and $C=0$ otherwise. The correlation coefficients at axial positions $z / D_{j} \approx 1,5,20$ and radial distances $r / r_{1 / 2} \approx 0,1,2$ are listed in Table 1 . In general, correlation improves downstream in the axial direction as turbulent mixing becomes more dominant. For a given axial position, the model performs best in the outer layer followed 


\begin{tabular}{c|ccc}
\hline$C\left(\bar{\tau}_{i j}, \tau_{i j}^{S G S}\right)$ & $r / r_{1 / 2} \approx 0$ & $r / r_{1 / 2} \approx 1$ & $r / r_{1 / 2} \approx 2$ \\
\hline$z / D_{j} \approx 1$ & 0.34 & 0.38 & 0.68 \\
$z / D_{j} \approx 5$ & 0.37 & 0.56 & 0.71 \\
$z / D_{j} \approx 20$ & 0.43 & 0.64 & 0.73 \\
\hline
\end{tabular}

Table 1: Correlation coefficient between $\bar{\tau}_{i j}$ and $\tau_{i j}^{S G S}$ at different axial and radial positions.

by the shear layer and worst in the centerline. The correlation coefficient provides a quantitative measure of the performance of the model. However, this measurement is very broad as it does not detail the rationale of the underlying differences.

\subsection{Eigenspace-based discrepancy}

The tensor eigendecompostion introduced in Section 3.2 offers a complimentary approach to the correlation coefficient. The discrepancy measurement is less compact since it does not provide a single scalar value, but it is potentially more informative as it allows one to separately analyze the differences in terms of magnitude, shape and orientation. This methodology is utilized next to further characterize the differences between $\bar{\tau}_{i j}$ and $\tau_{i j}^{S G S}$.

Focus is placed first on comparing the magnitude of the tensors as depicted in Figure 8 and summarized in Table 2. The plots correspond to probability density functions (PDF) of normalized $\tau_{k k} /\left(U_{j}-U_{e}\right)^{2}$ at different axial and radial positions. In general, for a given $z / D_{j}$, the mean of $\tau_{k k}$ is slightly larger at the shear layer than at the centerline of the jet. This trend is consistent for both reference and modeled tensors. However, the mean of the modeled tensor magnitude is increasingly underpredicted with respect to the reference as $z / D_{j}$ increases. The variance of $\tau_{k k}$ follows a similar behaviour. It is larger at $r / r_{1 / 2} \approx 1$ than at $r / r_{1 / 2} \approx 0$ and tends to be smaller for the modeled $\tau_{i j}$. The mode of the reference PDF is significantly well predicted by the model for $z / D_{j} \approx 1$ and for $z / D_{j} \approx 5$ at $r / r_{1 / 2} \approx 0$, while consistently underpredicted in the other locations by an approximate factor of 2 , viz. the model tends to predict $\tau_{k k}$ values in a smaller range on 
the left tail of the distributions.

The difference in anisotropy is analyzed next on the basis of the PDFs shown on the barycentric map for $\bar{\tau}_{i j}$ and $\tau_{i j}^{S G S}$ in Figures 9 and 10, respectively. From the $\bar{\tau}_{i j}$ perspective, the anisotropy of $\tau_{i j}$ in the centerline region remarkably evolves from the purely one-component limit at $z / D_{j} \approx 1$ to a wide PDF between axisymmetric expansion and the two-component limit at $z / D_{j} \approx 5,10$; the initial one-component shape is imposed by the walls of the pipe from which the jet discharges. This trend is similarly observed for $r / r_{1 / 2} \approx 1$, but less accentuated as the distribution at $z / D_{j} \approx 1$ is more stretched. A completely different behavior is depicted for $r / r_{1 / 2} \approx 2$. The mode of the PDF is initially located along the central region of the twocomponent limit, and with increasing $z / D_{j}$ it shifts toward a narrow distribution starting at the two-component vertex and following approximately the line of plane strain. The picture for $\tau_{i j}^{S G S}$ is notably different. At the centerline region, the shape of $\tau_{i j}^{S G S}$ is spread over the central and bottom regions of the barycentric map and it does not differ substantially between axial locations. The same anisotropy distribution is revealed for $r / r_{1 / 2} \approx 1$ at $z / D_{j} \approx 5,20$. For the remaining locations, the mode of the PDFs is found at the axisymmetric contraction limit close to the three-component vertex. The distributions are narrow and stretched along the axisymmetric contraction limit for $z / D_{j} \approx 1$, whereas they spread toward the central region of the barycentric map for $z / D_{j} \approx 5,20$. Further differences between filtered and modeled results can be extracted by considering the mean trajectories of $\tau_{i j}$ anisotropy in the axial direction at radial distances $r / r_{1 / 2} \approx 0,1$ represented in Figure 11. At the jet axis, $r / r_{1 / 2} \approx 0, \bar{\tau}_{i j}$ is completely one-component close to the nozzle, whereas it transitions, following an accelerating convex profile, to the central region of the barycentric map as $z / D_{j}$ increases. The behavior of $\tau_{i j}^{S G S}$ is substantially different as it starts from the central region of the triangle and becomes more spherical with increasing $z / D_{j}$ and displaying an almost vertical decelerating trajectory. At the jet's half-width 


\begin{tabular}{|c|c|c|c|c|c|c|}
\hline 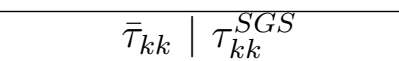 & \multicolumn{2}{|c|}{ Mean } & \multicolumn{2}{|c|}{ Variance } & \multicolumn{2}{|c|}{ Mode } \\
\hline$z / D_{j} \approx 1, r / r_{1 / 2} \approx 0$ & $4.4 \cdot 10^{-4}$ & $4.1 \cdot 10^{-4}$ & $5.5 \cdot 10^{-7}$ & $2.5 \cdot 10^{-7}$ & $1.3 \cdot 10^{-4}$ & $1.0 \cdot 10^{-4}$ \\
\hline$z / D_{j} \approx 1, r / r_{1 / 2} \approx 1$ & $4.5 \cdot 10^{-2}$ & $2.9 \cdot 10^{-2}$ & $3.3 \cdot 10^{-5}$ & $1.7 \cdot 10^{-5}$ & $6.5 \cdot 10^{-6}$ & $2.0 \cdot 10^{-6}$ \\
\hline$z / D_{j} \approx 5, r / r_{1 / 2} \approx 0$ & $1.7 \cdot 10^{-2}$ & $1.1 \cdot 10^{-2}$ & $3.5 \cdot 10^{-6}$ & $9.6 \cdot 10^{-7}$ & $1.0 \cdot 10^{-3}$ & $4.3 \cdot 10^{-4}$ \\
\hline$z / D_{j} \approx 5, r / r_{1 / 2} \approx 1$ & $4.1 \cdot 10^{-2}$ & $1.7 \cdot 10^{-2}$ & $9.4 \cdot 10^{-6}$ & $1.6 \cdot 10^{-6}$ & $1.2 \cdot 10^{-3}$ & $9.9 \cdot 10^{-4}$ \\
\hline$z / D_{j} \approx 20, r / r_{1 / 2} \approx 0$ & $5.1 \cdot 10^{-3}$ & $1.7 \cdot 10^{-3}$ & $1.3 \cdot 10^{-7}$ & $1.3 \cdot 10^{-8}$ & $1.8 \cdot 10^{-4}$ & $1.1 \cdot 10^{-4}$ \\
\hline$z / D_{j} \approx 20, r / r_{1 / 2} \approx 1$ & $7.7 \cdot 10^{-3}$ & $2.2 \cdot 10^{-3}$ & $3.6 \cdot 10^{-7}$ & $4.0 \cdot 10^{-8}$ & $4.9 \cdot 10^{-4}$ & $9.0 \cdot 10^{-5}$ \\
\hline
\end{tabular}

Table 2: PDF statistics of $\tau_{k k} /\left(U_{j}-U_{e}\right)^{2}$ (left: $\bar{\tau}_{k k}$, right: $\left.\tau_{k k}^{S G S}\right)$ at different axial and radial positions.

distance, $r / r_{1 / 2} \approx 1, \bar{\tau}_{i j}$ presents a similar initial and final states than at the jet axis, however, in this case the curve presents a convex shape. For $\tau_{i j}^{S G S}$, the behavior is completely different as it starts at the axisymmetric contraction limit. Interestingly, the curve ends at the central region of the map where it becomes similar to the $\bar{\tau}_{i j}$ anisotropy for $z / D_{j} \geq 20$.

Finally, the orientation of the tensors is analyzed by considering the eigenvector associated with the first eigenvalue of the eigendecomposition. The results (not shown) reveal a virtually perfect agreement between reference and modeled $\tau_{i j}$ eigenvectors since the orientation is directly imposed in the axial direction by the large scales of the jet. An interesting problem connected to this work would be the study of tensor alignments in a jet in crossflow (JICF) where the rapid tilting of the flow may impose sizable challenges to the SGS model from an orientation standpoint.

\subsection{Production of SGS kinetic energy discrepancy}

As discussed in Section 3.4.3, the anisotropy and orientation imposed on $\tau_{i j}^{S G S}$ in eddy-viscosity-type models force $\mathcal{P}_{r}$ to act as a sink of filtered kinetic energy. The dynamic approach [45] relaxes this constraint by allowing $\nu_{S G S}$ to take negative values in particular regions of the flow on the basis of the Germano identity [65] and a test-filtering operation. This methodology enables $\mathcal{P}_{r}$ to take negative values locally, and therefore it relatively accounts for backscatter effects. In terms of kinetic energy transport, as shown in Eq. 25, 
the negative sign of $\nu_{S G S}$ can be directly interpreted from an eigenspace perspective as a permutation between the first and third eigenvectors of the SGS stress tensor; namely, $\tau_{i j}^{S G S}$ and $\bar{S}_{i j}$ share the same eigenvalues and are rotated with respect to the first and third principal directions. In the case of utilizing the WALE SGS model, $\nu_{S G S}$ is dynamically calculated on the basis of the invariants of the velocity gradient tensor (recovering, for example, cubic behavior at walls [66]), however, defined always nonnegative by construction.

The discrepancy between normalized reference and modeled $\mathcal{P}_{r} /\left[\left(U_{j}-U_{e}\right)^{3} / D_{j}\right]$ at different axial and radial positions is depicted in Figure 12 and summarized in Table 3. An important observation is that the filtered numerical dataset exhibits significant amounts of backscatter (points of the PDF with $\left.\mathcal{P}_{r}<0\right)$ as indicated by the negative values of the PDF modes. This effect is not captured by the modeled $\mathcal{P}_{r}$ because of the aforementioned limitations of eddy-viscosity-type models as shown by the nonnegative distributions. In general, the mean of $\mathcal{P}_{r}$ is larger at $r / r_{1 / 2} \approx 1$ than at $r / r_{1 / 2} \approx 0$ and decreases with increasing $z / D_{j}$. This trend is consistent for both reference and modeled results. However, the mean $\mathcal{P}_{r}^{S G S}$ tends to be overestimated by a factor between 2 and 5 with respect to the filtered values. The spread of the reference and modeled $\mathcal{P}_{r}$ PDFs is qualitatively similar (except for the negative part). Nonetheless, the overall discrepancy in terms of $\mathcal{P}_{r}$ is not exceedingly large for the flow studied in this work. This is not typically the case in multiphysics flow problems in which small-scale phenomena impose significant misalignment between $\tau_{i j}$ and $\bar{S}_{i j}$, such as in combustion flames [67] and two-phase interfaces [68].

\section{Sensitivity analysis of model-form uncertainties}

The model-form uncertainty estimation framework developed also enables researchers to perform systematic sensitivity studies. Based on the discrepancies analyzed in Section 5, the impact of magnitude and shape model-form 


\begin{tabular}{c|cccc}
\hline$\overline{\mathcal{P}}_{r} \mid \mathcal{P}_{r}^{S G S}$ & \multicolumn{2}{|c}{ Mean } & Variance & Mode \\
\hline$z / D_{j} \approx 1, r / r_{1 / 2} \approx 0$ & $1.2 \cdot 10^{-4} \mid 5.6 \cdot 10^{-4}$ & $1.3 \cdot 10^{-7} \mid 2.2 \cdot 10^{-6}$ & $-2.9 \cdot 10^{-4} \mid 1.6 \cdot 10^{-6}$ \\
$z / D_{j} \approx 1, r / r_{1 / 2} \approx 1$ & $3.1 \cdot 10^{-3} \mid 1.5 \cdot 10^{-2}$ & $9.4 \cdot 10^{-5} \mid 1.8 \cdot 10^{-3}$ & $-3.2 \cdot 10^{-2} \mid 1.6 \cdot 10^{-5}$ \\
$z / D_{j} \approx 5, r / r_{1 / 2} \approx 0$ & $9.3 \cdot 10^{-4} \mid 2.0 \cdot 10^{-3}$ & $8.7 \cdot 10^{-6} \mid 1.6 \cdot 10^{-5}$ & $-4.2 \cdot 10^{-3} \mid 8.8 \cdot 10^{-6}$ \\
$z / D_{j} \approx 5, r / r_{1 / 2} \approx 1$ & $1.9 \cdot 10^{-3} \mid 2.9 \cdot 10^{-3}$ & $1.8 \cdot 10^{-5} \mid 2.4 \cdot 10^{-5}$ & $-1.8 \cdot 10^{-2} \mid 2.3 \cdot 10^{-3}$ \\
$z / D_{j} \approx 20, r / r_{1 / 2} \approx 0$ & $7.9 \cdot 10^{-5} \mid 7.5 \cdot 10^{-5}$ & $3.9 \cdot 10^{-8} \mid 1.0 \cdot 10^{-8}$ & $-6.3 \cdot 10^{-4} \mid 4.2 \cdot 10^{-6}$ \\
$z / D_{j} \approx 20, r / r_{1 / 2} \approx 1$ & $6.1 \cdot 10^{-5} \mid 6.8 \cdot 10^{-5}$ & $3.0 \cdot 10^{-8} \mid 3.1 \cdot 10^{-8}$ & $-1.3 \cdot 10^{-3} \mid 2.1 \cdot 10^{-5}$ \\
\hline
\end{tabular}

Table 3: PDF statistics of $\mathcal{P}_{r} /\left[\left(U_{j}-U_{e}\right)^{3} / D_{j}\right]\left(\right.$ left: $\overline{\mathcal{P}}_{r}$, right: $\left.\mathcal{P}_{r}^{S G S}\right)$ at different axial and radial positions.

uncertainties on the QoIs are examined. These two uncertainties consider three of the six degrees of freedom of $\tau_{i j}$ and are independently related to $\mathcal{P}_{r}$ through $\nu_{S G S}$ in the case of eddy-viscosity-type closures (magnitude) and the sum of $\lambda_{i}-\gamma_{i}$ products (anisotropy) as shown in Eqs. 24 and 25. Upon selection of the WALE SGS closure as the base model, propagation of incertitude in the magnitude of $\tau_{i j}^{S G S}$ is studied by augmenting and decreasing $\tau_{k k}$ as proposed by the maximum and minimum limits of the perturbation defined in Eq. 19. As illustrated in Figure 13, model-form uncertainty in the spectrum of $\tau_{i j}^{S G S}$ is analyzed by perturbing the eigenvalues of the base model tensor toward the three vertices of the barycentric map with relative distance $\Delta_{B}=5 \%$. Complete implementation details on how to apply the UQ framework in a general LES solver are provided in the Appenidx. The shaded regions in Figures 14-18 depict the envelope of predictions resulting from the perturbation UQ estimation; i.e., minimim and maximum bounds of the predictions provided by the ensemble set of 6 calculations (base WALE model, $\Delta \tau_{k k}^{S G S} \leq 0$ and $\Delta \tau_{k k}^{S G S} \geq 0$ magnitude perturbations, anisotropy perturbations toward vertexes $\mathbf{x}_{1 c}, \mathbf{x}_{2 c}$ and $\mathbf{x}_{3 c}$ ). In general, the uncertainty estimates adaptively envelope the reference data for most of the profiles, displaying wider regions at points where the base model significantly deviates from the reference solution. Details of the results for the different QoIs are discussed below. 


\subsection{Time-averaged flow quantities}

Results of uncertainty estimates for mean axial velocity profiles are depicted in Figure 14. The shaded regions, representative of the uncertainty perturbation solutions, clearly envelope the reference data along the jet axis and radial profiles at different axial positions. Moreover, the width of the envelopes broadens in regions where discrepancy between the WALE model and reference data predictions increases, i.e., $z / D_{j}>5$ and $r / r_{1 / 2}<2$, whereas it narrows away from the axis where the turbulence activity is lower. For these plots, the upper and lower bounds correspond to the solutions obtained by reducing the trace of the tensor and by perturbing the eigenvalues toward vertex $\mathbf{x}_{1 c}$ of the barycentric map, respectively. The performance of the perturbation UQ framework, in terms of enveloping the reference data, is similar for the normal and shear stresses, and the aggregate turbulent kinetic energy, shown in Figures 15-18, except for $z / D_{j} \approx 1$, in which the numerical dataset is not covered by the space of perturbed solutions for $1<r / r_{1 / 2}<2$. In this region, the flow field is significantly dominated by the inflow boundary conditions, with the SGS model not playing an important role. A common observation for the uncertainty estimates of the second-order statistics is that the width of the shaded areas slightly increases with $z / D_{j}$. In addition, the bounds of the envelopes display a general change of trend: the upper and lower bounds for $r / r_{1 / 2}<1$ result from augmenting the trace and forcing the SGS stresses to be more rod-like, while perturbing toward a rod-like shape and reducing the magnitude provide the upper and lower bounds for $r / r_{1 / 2}>1$. In general, the first- and second-order QoIs studied are sensitive to reducing the magnitude and increasing the anisotropy of $\tau_{i j}^{S G S}$ in one direction. On the contrary, they are strongly independent to the other perturbations considered: increase of tensor magnitude and anisotropy perturbation toward two- and three-component vertices of the barycentric map.

A detailed analysis of the impact of $\tau_{i j}^{S G S}$ model-form uncertainty on 
the Reynolds shear-stress and turbulent kinetic energy is presented in Figures 19 and 20, where the axial and radial distributions of these quantities on the $x$ - $z$ azimuthal plane is depicted for the (a) numerical reference dataset, (b) WALE SGS model, (c) WALE SGS model with $\Delta \tau_{k k}^{S G S} \leq 0$ magnitude perturbation, and (d) WALE SGS model with anisotropy perturbation toward vertex $\mathbf{x}_{1 c}$. In comparison to the reference solution, the WALE SGS model overpredicts the thickness of the shear layer and the magnitude of the stresses approximately by $2 \times$ in the region $1<z / D_{j}<10$, resulting in a shorter potential core, while it performs similarly to the reference dataset away from the nozzle, $z / D_{j}>10$, where the turbulent flow becomes more isotropic. In the case of reducing the trace of $\tau_{i j}^{S G S}$, the effects are roughly inversed; viz. shear stresses are overpredicted for $z / d_{j}<1$, whereas both the intensity (slightly) and thickness of the shear layer are underestimated in $1<z / D_{j}<10$. Similarly to the WALE SGS model, perturbation of $\tau_{i j}^{S G S}$ toward a more rod-like shape results in a moderate overestimation of the magnitude of the Reynolds shear-stress in the region $1<z / D_{j}<10$ and the thickness of the shear layer for all $z / D_{j}$. In terms of turbulent kinetic energy, the WALE SGS model produces results displaying faster mixing with a shorter potential core than the reference as it can be seen by the larger spread of TKE in the region $1<z / D_{j}<10$. On the contrary, reducing the trace of $\tau_{i j}^{S G S}$ provides a solution similar to the numerical dataset for $z / D_{j}>1$, but presenting smaller values of TKE in the axis of the jet which is indicative of a longer survival of the potential core. Perturbing the shape of $\tau_{i j}^{S G S}$ toward the one-component limit produces a potential core similar to the reference dataset and with comparable TKE layer thickness for $z / D_{j}<1$, but presenting increased entrainment in the region $1<z / D_{j}<10$ as observed by the larger extension of the TKE band on the external side.

The rationality behind the perturbations responsible for the upper and lower bounds of the second-order statistics can be related to filtered kinetic energy arguments. The WALE model tends to generally underpredict the 
numerical dataset. Therefore, the WALE model solutions can already be interpreted as a lower bound, since decreasing the magnitude of the tensor provides even lower predictions. Per contra, perturbations to the shape of the tensor toward the one-component vertex of the barycentric map generates, in general, the upper bound of the uncertainty estimation envelopes. This large sensitivity to anisotropy perturbation is consistent with the discrepancy observed by comparing Figures 9 and 10. The reference dataset depicts $\tau_{i j}$ anisotropies in the one-component vertex region for $r / r_{1 / 2} \approx 0,1$, while the WALE model predicts shapes in the central region of the triangle and toward the axisymmetric contraction limit. Consequently, applying perturbations in the one-component vertex direction forces the WALE model to produce SGS stresses more aligned with the reference dataset observations which result in an upper bound. In terms of production of SGS kinetic energy, the following mathematical expression is obtained for the UQ framework based on an eddyviscosity model without eigenvector perturbation

$$
\mathcal{P}_{r}^{*}=2 \nu_{S G S} \frac{\tau_{k k}^{S G S^{*}}}{\tau_{k k}^{S G S}}\left(\lambda_{1}^{*} \gamma_{1}+\lambda_{2}^{*} \gamma_{2}+\lambda_{3}^{*} \gamma_{3}\right)
$$

Therefore, if the trace of the tensor is reduced, i.e., $\tau_{k k}^{S G S^{*}}<\tau_{k k}^{S G S}, \mathcal{P}_{r}^{*}=$ $2 \nu_{S G S} \tau_{k k}^{S G S^{*}} / \tau_{k k}^{S G S}\left(\gamma_{1}^{2}+\gamma_{2}^{2}+\gamma_{3}^{2}\right)$ decreases, and consequently the flow field contains more filtered kinetic energy as a result of smaller forward-scatter rates, which eventually leads to a slower decay of the potential core. An opposite effect is obtained when applying the perturbation toward the onecomponent vertex since $\mathcal{P}_{r}^{*}=2 \nu_{S G S}\left(\lambda_{1}^{*} \gamma_{1}+\lambda_{2}^{*} \gamma_{2}+\lambda_{3}^{*} \gamma_{3}\right)$ tends to augment due to the larger magnitude of the first eigenvalue relative to the other anisotropy states. This increase in $\mathcal{P}_{r}^{*}$ drains more rapidly kinetic energy from the large to the SGS scales, slightly laminarizing the flow and accelerating the disintegration of the potential core. 


\subsection{Instantaneous flow quantities}

The conclusions extracted from time-averaged statistics are also observed from instantaneous flow quantities. These can be inferred, for instance, from Figures 21-23 which show several snapshots of normalized $x y$-plane and $z$ resolved vorticity, i.e., $\left(\bar{\omega}_{x}^{2}+\bar{\omega}_{y}^{2}\right)^{1 / 2} /\left[\left(U_{j}-U_{e}\right) / D_{j}\right]$ and $\bar{\omega}_{z} /\left[\left(U_{j}-U_{e}\right) / D_{j}\right]$, and $r z$ resolved rate-of-strain, $\bar{S}_{r z} /\left[\left(U_{j}-U_{e}\right) / D_{j}\right]$, at axial cross sections $z / D_{j}=1,5,10$ for the reference numerical dataset, WALE SGS model, WALE SGS model with $\Delta \tau_{k k}^{S G S} \leq 0$ magnitude perturbation, and WALE SGS model with anisotropy perturbation toward vertex $\mathbf{x}_{1 c}$.

The initial circular shear layer increasingly develops larger wrinkles as the flow moves downstream. As shown by the reference dataset in Figures 21 and 22 , these corrugations result in vorticity generation that spreads perpendicularly to the jet axis and mixes with the surrounding flow while reducing its magnitude. In comparison to the reference vorticity distribution, the WALE SGS model and anisotropy perturbation toward one-component (especially) predict fewer larger vortical structures, whereas reducing the trace of $\tau_{i j}^{S G S}$ results in an increase of number of vortexes presenting smaller sizes and enhanced mixing. In the case of the WALE SGS model, these features are especially noticeable for $z / D_{j}=1$, while are clearly observable at all distances for the case of perturbing toward one-component. In other words, decreasing the magnitude of the SGS stresses propitiates the creation of small scales resulting from an increased fragmentation of the vortex rings created at the shear layer of the jet. This phenomenon leads to a shorter potential core as a result of increased flow mixing.

Equivalent trends are recognized in Figure 23 for the normalized, resolved $r z$ rate-of-strain. As displayed by the numerical reference, the radial deformation rate of the flow generally reduces in time as it moves downstream in the axial direction, viz. dominance of negative-valued regions. This characteristic is well captured by the three LES exhibited. However, they present large differences in terms of magnitude and spatial distribution. Similarly 
to resolved vorticity, slightly perturbing $\tau_{i j}^{S G S}$ toward one-component has a significant impact on the deformation rate of the large scales. Particularly, $\bar{S}_{r z}$ becomes more negatively dominated and continuous along the circular region of the jet's shear layer, indicating that the flow undergoes lesser deformation in the radial direction downstream the nozzle which propitiates a longer survival of the potential core by means of a diminished shear layer. The contrary is observed when reducing the magnitude of the SGS stresses. In that case, $\bar{S}_{r z}$ presents larger amounts of positive regions combined with higher levels of fragmentation, especially downstream in the axial direction.

\section{Conclusions}

An eigensensitivity analysis of SGS model-form uncertainty has been performed on a LES of a round turbulent jet. Experimental and numerical reference data have been utilized to validate the observations of the study in terms of averaged and rms axial and radial velocities, shear stresses and turbulent kinetic energy. The numerical reference dataset has been generated by carrying out highly accurate (DNS-like resolution) simulations based on the setup of the reference experiment. Focus has been placed on QoIs at the jet's centerline and half-width for different axial distances, as these correspond to regions of the flow characterizing the potential core and exhibiting maximum production of turbulent kinetic energy, respectively. Complete agreement between the reference datasets has been obtained for first- and (virtually) second-order flow statistics.

Differences in statistics between the numerical reference solution and a LES based on the eddy-viscosity WALE SGS model have been observed for the averaged axial velocity along the jet axis in the interaction region and, more significantly, for second-order flow quantities in $r / r_{1 / 2}<1$. A priori eigendecomposition analyses of differences between reference and modeled SGS stress tensors in terms of magnitude, shape and orientation have shown potential sources of discrepancy. Three main observations have been ex- 
tracted: (i) in general, the magnitude of the modeled tensor tends to be slightly underpredicted with respect to the reference SGS stresses, (ii) the correlation between tensors in terms of anisotropy is outstandingly low as the reference tensor tends to lie close to the one-component vertex and twocomponent limit of the barycentric map while the PDF of the modeled tensor is concentrated in the central region and axisymmetric contraction limit, (iii) the alignment of the principal directions in the reference and modeled tensors presents notable agreement. Consequently, from a general LES modeling point of view, the discrepancies identified in this work indicate that improved eddy-viscosity-type SGS models for shear-dominated flows, rather than focusing on modifying the turbulent viscosity, should consider modeling approaches in which SGS anisotropy is better represented.

On the basis of the a priori discrepancy observations, the impact of magnitude and anisotropy model-form uncertainty on different QoIs have been a posteriori analyzed. In terms of time-averaged flow quantities, the general observation is that the uncertainty estimates adaptively envelope the reference data, displaying wider regions at points where the base model significantly deviates from the reference solution. The reduction of SGS stresses' magnitude and perturbation toward one-component anisotropy provide the larger impacts on flow statistics. Perturbations to the shape of the tensor present, in general, larger relative impact than reducing, or augmenting, the magnitude of the tensor; similar order deviations are observed for both types of discrepancies, but the perturbations related to shape discrepancy are relatively small $(5 \%)$ compared with the $\mathcal{O}(1)$ magnitude perturbations. A common observation for the uncertainty estimates is that the width of the envelopes slightly increases with axial distance, indicating that modelform uncertainty is characterized by a cumulative behaviour in free shear flows. The effect of SGS stresses discrepancy on flow quantities has been analyzed also by means of qualitative visualizations of instantaneous, spatial vorticity and rate-of-strain distributions. In comparison to the reference 
vorticity, the base model and anisotropy perturbation toward one-component predict fewer larger vortical structures, whereas reducing the trace of the SGS stresses results in an increase of number of vortexes presenting smaller sizes and enhanced mixing. Equivalent trends are observed for the rate-of-strain since slightly perturbing the SGS stresses toward one-component results in negatively dominated and continuous rate-of-strain structures along the circular region of the jet's shear layer, indicating that the flow undergoes lesser deformation in the radial direction downstream the nozzle which propitiates a longer survival of the potential core by means of a diminished shear layer. The contrary is observed when reducing the magnitude of the SGS stresses.

In a more general perspective, the UQ framework presented to characterize model-form sensitivity to SGS stress modeling has been shown to be an effective approach for the efficient and systematic exploration/study of complex flow phenomena by means of predictive LES. Ongoing work is focused on eigensensitivity analyses of SGS model-form uncertainty in variabledensity free shear flows. Additionally, studies of the impact on the QoIs of mixed uncertainties involving, for example, SGS model-form and aleatoric incertitude on turbulent jets in crossflow are being conducted. Future work will concentrate on developing transport equations for the parameters of the methodology such that injection of incertitude is restricted to regions of the flow where the SGS models are expected to provide less accurate predictions.

\section{Acknowledgments}

This investigation was funded by the Advanced Simulation and Computing (ASC) program of the US Department of Energy's National Nuclear Security Administration via the PSAAP-II Center at Stanford University, Grant No. DE-NA0002373.

Sandia National Laboratories is a multi-mission laboratory managed and operated by National Technology and Engineering Solutions of Sandia, LLC., a wholly owned subsidiary of Honeywell International, Inc., for the U.S. De- 
partment of Energy's National Nuclear Security Administration under contract DE-NA-0003525. This paper describes objective technical results and analysis. Any subjective views or opinions that might be expressed in the paper do not necessarily represent the views of the U.S. Department of Energy or the United States Government. SAND2019-0078 J.

The authors would like to thank the anonymous reviewers for their valuable comments and suggestions to help improve the quality of the paper.

\section{Appendix A: Framework implementation overview}

The uncertainty quantification framework described in this work is developed with the objective of being suitable to LES solvers in complex geometries. A general example would be, for instance, the unstructured and massively parallel Nalu open-source code [59, 69] utilized in the numerical experiments section. For this purpose, an implementation overview of the framework is described below.

Similar to the calculation of the turbulent viscosity in eddy-viscosity-type models, introduction of the perturbations is performed locally at each time step. Therefore, the framework is inherently parallel and easy to implement on 3-D unstructured meshes. For a general combination of perturbations, four main steps are required.

The first step is to construct $a_{i j}^{S G S}$ from the base-model definition. For example, in the case of eddy-viscosity models, $\overline{u_{k} u_{k}}$ and $-2 \nu_{S G S} \bar{S}_{i j}$ need to be calculated. The latter is directly accessible in most LES solvers as $\nu_{S G S}$ is typically evaluated from expressions involving $\bar{S}_{i j}$. The former, however, is less commonly available since it requires modeling $\tau_{k k}^{S G S}$.

Step number two is to perform the spectral decomposition of $a_{i j}^{S G S}$. Many efficient and robust methods exist for $3 \times 3$ symmetric matrices. For instance, optimized algorithms can be found in [70]. Once the eigendecomposition is obtained, the eigenvalues and the corresponding eigenvectors need to be sorted such that $\lambda_{1}^{S G S} \geq \lambda_{2}^{S G S} \geq \lambda_{3}^{S G S}$ is satisfied. 
The following step, number three, is to apply perturbations (individual or a combination) to $a_{i j}^{S G S}$ within the framework described in Sec. 3. Next, the perturbed decomposition is reassembled to generate $a_{i j}^{S G S^{*}}=v_{i n}^{S G S^{*}} \Lambda_{n l}^{S G S^{*}} v_{j l}^{S G S^{*}}$. Finally, in step number four, $a_{i j}^{S G S^{*}}$ is multiplied by ${\overline{u_{k} u_{k}}}^{*}$, and the divergence of the resulting tensor, $\overline{u_{k} u_{k}}{ }^{*} a_{i j}^{S G S^{*}}$, is introduced into the LES equations. Notice that

$$
{\overline{u_{i} u_{j}}}^{*}=\bar{u}_{i} \bar{u}_{j}+\tau_{i j}^{S G S^{*}}=\bar{u}_{i} \bar{u}_{j}+{\overline{u_{k} u_{k}}}^{*} a_{i j}^{S G S^{*}}+\frac{\tau_{k k}^{S G S^{*}}}{3} \delta_{i j} .
$$

Therefore, instead of augmenting the molecular viscosity, $\nu$, with the turbulent viscosity, $\nu_{S G S}$, as it is typical in most LES solvers, the SGS term in this framework is treated independently from the viscous stresses since the eigenvalues and eigenvectors of $\bar{S}_{i j}$ and $a_{i j}^{S G S^{*}}$ are generally different after the perturbations are applied. The isotropic term $\tau_{k k}^{S G S^{*}} / 3$ should be computed and integrated into the equations for compressible flows, while it can be absorbed into the filtered pressure when considering incompressible flow.

\section{References}

[1] U. Piomelli, E. Balaras, Wall-layer models for large-eddy simulations, Annu. Rev. Fluid Mech. 34 (2002) 349-374.

[2] R. O. Fox, Large-eddy-simulation tools for multiphase flows, Annu. Rev. Fluid Mech. 44 (2012) 47-76.

[3] J. G. M. Kuerten, Point-particle DNS and LES of particle-laden turbulent flow - a state-of-the-art review, Flow Turbul. Combust. 97 (2016) 689-713.

[4] J. Smagorinsky, General circulation experiments with the primitive equations. I. The basic experiment, Mon. Weather Rev. 91 (1963) 99164. 
[5] F. Porté-Agel, C. Meneveau, M. B. Parlange, A scale-dependent dynamic model for large-eddy simulation: application to a neutral atmospheric boundary layer, J. Fluid Mech. 415 (2000) 261-284.

[6] H. Pitsch, Large-eddy simulation of turbulent combustion, Annu. Rev. Fluid Mech. 38 (2006) 453-482.

[7] M. Masquelet, J. Yan, A. Dord, G. Laskowski, L. Shunn, L. Jofre, G. Iaccarino, Uncertainty quantification in large eddy simulations of a rich-dome aviation gas turbine, in: Proc. ASME Turbo Expo 2017, GT2017-64835, pp. 1-11.

[8] S. B. Pope, Turbulent Flows, Cambridge University Press, 2000.

[9] H. Tennekes, J. L. Lumley, A first course in turbulence, The MIT Press, 1972.

[10] S. Cheung, T. Oliver, E. Prudencion, S. Pridhomme, R. Moser, Bayesian uncertainty analysis with applications to turbulence modeling, Reliab. Eng. Syst. Safe 96 (2011) 1137-1149.

[11] C. Meneveau, J. Katz, Scale-invariance and turbulence models for largeeddy simulation, Annu. Rev. Fluid Mech. 32 (2000) 1-32.

[12] R. A. Clark, J. H. Ferziger, W. C. Reynolds, Evaluation of subgrid-scale models using an accurately simulated turbulent flow, J. Fluid Mech. 91 (1979) 1-16.

[13] J. Bardina, J. H. Ferziger, W. C. Reynolds, Improved subgrid scale models for large eddy simulation, in: Proc. AIAA 13th Fluid \& Plasma Dynamics Conference, pp. 1-10.

[14] Y. Zang, R. L. Street, J. R. Koseff, A dynamic mixed subgrid-scale model and its application to turbulent recirculating flows, Phys. Fluids A 5 (1993) 3186-3195. 
[15] S. Ghosal, An analysis of numerical errors in large-eddy simulations of turbulence, J. Comput. Phys. 125 (1996) 187-206.

[16] J. Meyers, B. J. Geurts, M. Baelmans, Database analysis of errors in large-eddy simulation, Phys. Fluids 15 (2003) 2740.

[17] M. Meldi, D. Lucor, P. Sagaut, Is the Smagorinsky coefficient sensitive to uncertainty in the form of the energy spectrum?, Phys. Fluids 23 (2011) 125109.

[18] J. Meyers, P. Sagaut, Evaluation of Smagorinsky variants in large-eddy simulations of wall-resolved plane channel flows, Phys. Fluids 19 (2007) 095105 .

[19] J. Meyers, P. Sagaut, Is plane-channel flow a friendly case for the testing of large-eddy simulation subgrid-scale models?, Phys. Fluids 19 (2007) 048105.

[20] M. C. Dunn, B. Shotorban, A. Frendi, Uncertainty quantification of turbulence model coefficients via Latin hypercube sampling method, J. Fluids Eng. 133 (2011) 041402.

[21] D. Lucor, J. Meyers, P. Sagaut, Sensitivity analysis of large-eddy simulations to subgrid-scale-model parametric uncertainty using polynomial chaos, J. Fluid Mech. 585 (2007) 255-280.

[22] S. Völker, R. Moser, P. Venugopal, Optimal large eddy simulation of turbulent channel flow based on direct numerical simulation statistical data, Phys. Fluids 14 (2002) 3675-3691.

[23] W. N. Edeling, P. Cinnella, R. P. Dwight, Bayesian estimates of parameter variability in the $k-\epsilon$ turbulence model, J. Comput. Phys. 258 (2014) 73-94. 
[24] C. Safta, M. Blaylock, J. Templeton, S. Domino, K. Sargsyan, H. Njam, Uncertainty quantification in LES of channel flow, Int. J. Numer. Meth. Fluids 83 (2017) 376-401.

[25] N. A. Phillips, Models for weather prediction, Annu. Rev. Fluid Mech. 2 (1970) 251-292.

[26] C. E. Leith, Objective methods for weather prediction, Annu. Rev. Fluid Mech. 10 (1978) 107-128.

[27] B. Stevens, C.-H. Moeng, A. S. Ackerman, C. S. Bretherton, A. Chlond, S. de Roode, J. Edwards, J.-C. Golaz, H. Jiang, M. Khairoutdinov, M. P. Kirkpatrick, D. C. Lewellen, A. Lock, F. M uller, D. Stevens, E. Whelan, P. Zhu, Evaluation of large-eddy simulations via observations of nocturnal marine stratocumulus, Mon. Weather Rev. 133 (2005) 1443-1462.

[28] L. Jofre, S. P. Domino, G. Iaccarino, A framework for characterizing structural uncertainty in large-eddy simulation closures, Flow Turbul. Combust. 100 (2018) 341-363.

[29] C. Gorlé, G. Iaccarino, A framework for epistemic uncertainty quantification of turbulent scalar flux models for Reynolds-averaged NavierStokes simulations, Phys. Fluids 25 (2013) 055105.

[30] M. Emory, J. Larsson, G. Iaccarino, Modeling of structural uncertainties in Reynolds-averaged Navier-Stokes closures, Phys. Fluids 25 (2013) 110822.

[31] G. Iaccarino, A. A. Mishra, S. Ghili, Eigenspace perturbations for uncertainty estimation of single-point turbulence closures, Phys. Rev. Fluids 2 (2017) 024605.

[32] E. J. Parish, K. Duraisamy, A paradigm for data-driven predictive modeling using field inversion and machine learning, J. Comput. Phys. 305 (2016) 758-774. 
[33] H. Xiao, J.-L. Wu, J.-X. Wang, R. Sun, C. J. Roy, Quantifying and reducing model-form uncertainties in Reynolds-averaged Navier-Stokes simulations: A data-driven, physics-informed Bayesian approach, J. Comput. Phys. 324 (2016) 115-136.

[34] J. Ling, A. Kurzawski, J. Templeton, Reynolds averaged turbulence modelling using deep neural networks with embedded invariance, J. Fluid Mech. 807 (2016) 155-166.

[35] M. Olsson, L. Fuchs, Large eddy simulation of the proximal region of a spatially developing circular jet, Phys. Fluids 8 (1996) 2125.

[36] C. Bogey, C. Bailly, Large eddy simulations of transitional round jets: Influence of the Reynolds number on flow development and energy dissipation, Phys. Fluids 18 (2006) 065101.

[37] P. Wang, J. Fr ohlich, V. Michelassi, W. Rodi, Large-eddy simulation of variable-density turbulent axisymmetric jets, Int. J. Heat Fluid Flow 29 (2008) 654-664.

[38] C. Bogey, C. Bailly, Turbulence and energy budget in a self-preserving round jet: direct evaluation using large eddy simulation, J. Fluid Mech. 627 (2009) 129-160.

[39] J. Kim, H. Choi, Large eddy simulation of a circular jet: effect of inflow conditions on the near field, J. Fluid Mech. 620 (2009) 383-411.

[40] O. V. Vasilyev, T. S. Lund, P. Moin, A general class of commutative filters for LES in complex geometries, J. Comput. Phys. 146 (1998) 82-104.

[41] A. L. Marsden, O. V. Vasilyev, P. Moin, Construction of commutative filters for LES on unstructured meshes, J. Comput. Phys. 175 (2002) 584-603. 
[42] A. Leonard, Energy cascade in large-eddy simulations of turbulent fluid flows, Adv. Geophys. A 18 (1974) 237-248.

[43] T. S. Lund, The use of explicit filters in large eddy simulation, Comput. Math. Appl. 46 (2003) 603-616.

[44] R. S. Rogallo, P. Moin, Numerical simulation of turbulent flow, Annu. Rev. Fluid Mech. 16 (1984) 2150.

[45] M. Germano, U. Piomelli, P. Moin, W. Cabot, A dynamic subgrid-scale eddy viscosity model, Phys. Fluids A 3 (1991) 1760-1765.

[46] F. Nicoud, F. Ducros, Subgrid-scale stress modelling based on the square of the velocity gradient, Flow Turbul. Combust. 62 (1999) 183-200.

[47] F. Nicoud, H. B. Toda, O. Cabrit, S. Bose, J. Lee, Using singular values to build a subgrid-scale model for large eddy simulations, Phys. Fluids 23 (2011) 085106.

[48] W. Rozema, H. J. Bae, P. Moin, R. Verstappen, Minimum-dissipation models for large-eddy simulation, Phys. Fluids 27 (2015) 085107.

[49] A. Yoshizawa, Statistical theory for compressible turbulent shear flows, with the application to subgrid modeling, Phys. Fluids 29 (1986) 21522164.

[50] P. Moin, K. Squires, W. Cabot, S. Lee, A dynamic subgrid-scale model for compressible turbulence and scalar transport, Phys. Fluids A 3 (1991) 2746-2757.

[51] B. Vreman, B. Geurts, H. Kuerten, Realizability conditions for the turbulent stress tensor in large-eddy simulation, J. Fluid Mech. 278 (1994) 351-362.

[52] C. G. Speziale, Galilean invariance of subgrid-scale stress models in the large-eddy simulation of turbulence, J. Fluid Mech. 156 (1985) 55-62. 
[53] S. Banerjee, R. Krahl, F. Durst, C. Zenger, Presentation of anisotropy properties of turbulence, invariants versus eigenvalues approaches, J. Turbul. 8 (2007) 1-27.

[54] G. Kindlmann, Superquadric tensor glyphs, in: Proc. 6th Joint Eurographics-IEEE TCVG Conference, pp. 147-154.

[55] Teem, Tools to process and visualize scientific data and images, 2003.

[56] U. Piomelli, W. Cabot, P. Moin, S. Lee, Subgrid-scale backscatter in turbulent and transitional flows, Phys. Fluids 3 (1991) 1766-1771.

[57] J. B. Lasserre, A trace inequality for matrix product, IEEE Trans. Autom. Control 40 (1995) 1500-1501.

[58] M. Amielh, T. Djeridane, F. Anselmet, L. Fulachier, Velocity near-field of variable density turbulent jets, Int. J. Heat Mass Transfer 39 (1996) 2149-2164.

[59] S. P. Domino, Sierra Low Mach Module: Nalu Theory Manual 1.0, Technical Report SAND2015-3107W, Sandia National Laboratories, Unclassified Unlimited Release (UUR), 2015.

[60] S. P. Domino, Design-order, non-conformal low-Mach fluid algorithms using a hybrid CVFEMDG, J. Comput. Phys. 359 (2018) 331-351.

[61] S. P. Domino, P. Sakievich, M. Barone, An assessment of atypical mesh topologies for low-Mach large-eddy simulation, Comput. Fluids 179 (2019) 655-669.

[62] B. J. Cantwell, Introduction to Symmetry Analysis, Cambridge University Press, 2002.

[63] C. B. da Silva, J. C. F. Pereira, Invariants of the velocity-gradient, rate-of-strain, and rate-of-rotation tensors across the turbulent/nonturbulent interface in jets, Phys. Fluids 20 (2008) 055101. 
[64] P. Sagaut, R. Grohens, Discrete filters for large eddy simulation, Int. J. Numer. Meth. Fluids 31 (1999) 1195-1220.

[65] M. Germano, Turbulence: the filtering approach, J. Fluid Mech. 238 (1992) 325-336.

[66] D. Chapman, G. Kuhn, The limiting behavior of turbulence near a wall, J. Fluid Mech. 170 (1986) 265-292.

[67] J. O'Brien, J. Urzay, M. Ihme, P. Moin, A. Saghafian, Subgrid-scale backscatter in reacting and inert supersonic hydrogen-air turbulent mixing layers, J. Fluid Mech. 743 (2014) 554-584.

[68] M. S. Dodd, L. Jofre, Tensor-based analysis of the flow topology in droplet-laden homogeneous isotropic turbulence, Annual Research Briefs, Center for Turbulence Research, Stanford University (2018) 3545 .

[69] S. P. Domino, L. Jofre, G. Iaccarino, The suitability of hybrid meshes for low-Mach large-eddy simulation, Proceedings of the Summer Program, Center for Turbulence Research, Stanford University (2018) 97-106.

[70] W. H. Press, S. A. Teukolsky, W. T. Vetterling, B. P. Flannery, Numerical recipes, Cambridge University Press, 2007. 

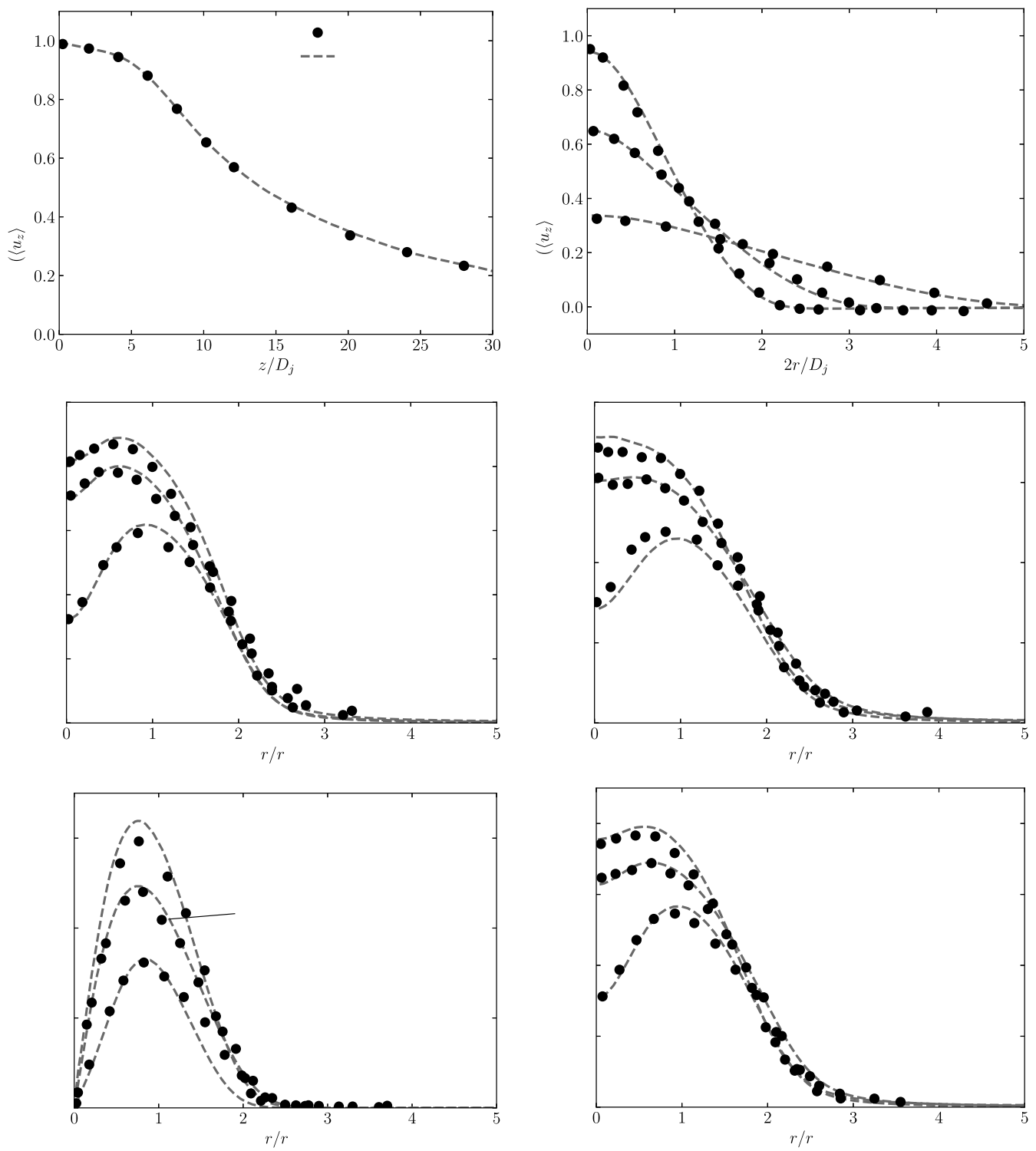

Figure 5: Comparison of the numerical dataset against experimental data by Amielh et al. [58]. (a) Mean axial velocity along the jet axis. (b) Radial profiles of mean axial velocity at several axial positions. (c) Radial profiles of rms axial velocity at different axial positions. (d) Radial profiles of rms radial velocity at different axial positions. (e) Radial profiles of Reynolds shear stress at different axial positions. (f) Radial profiles of turbulent kinetic energy at different axial positions. 

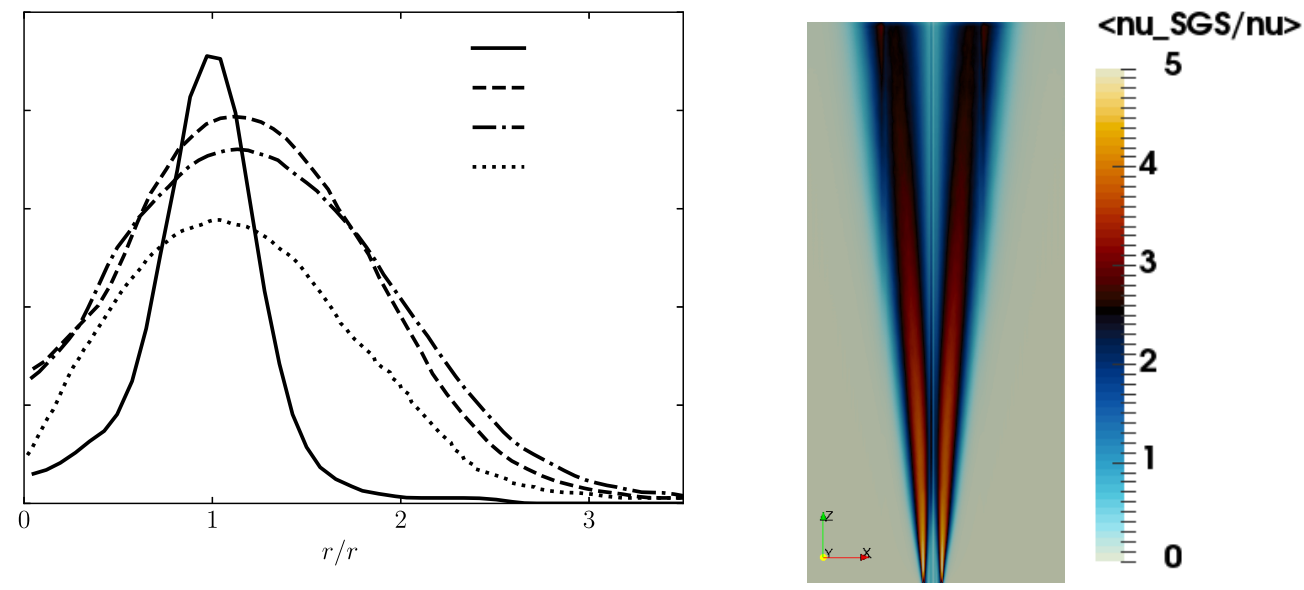

Figure 6: Ratio of averaged turbulent viscosity, $\nu_{S G S}$, to kinematic viscosity, $\nu$. (left) Radial profiles at different axial positions. (right) Visualization on the $x z$-plane. 

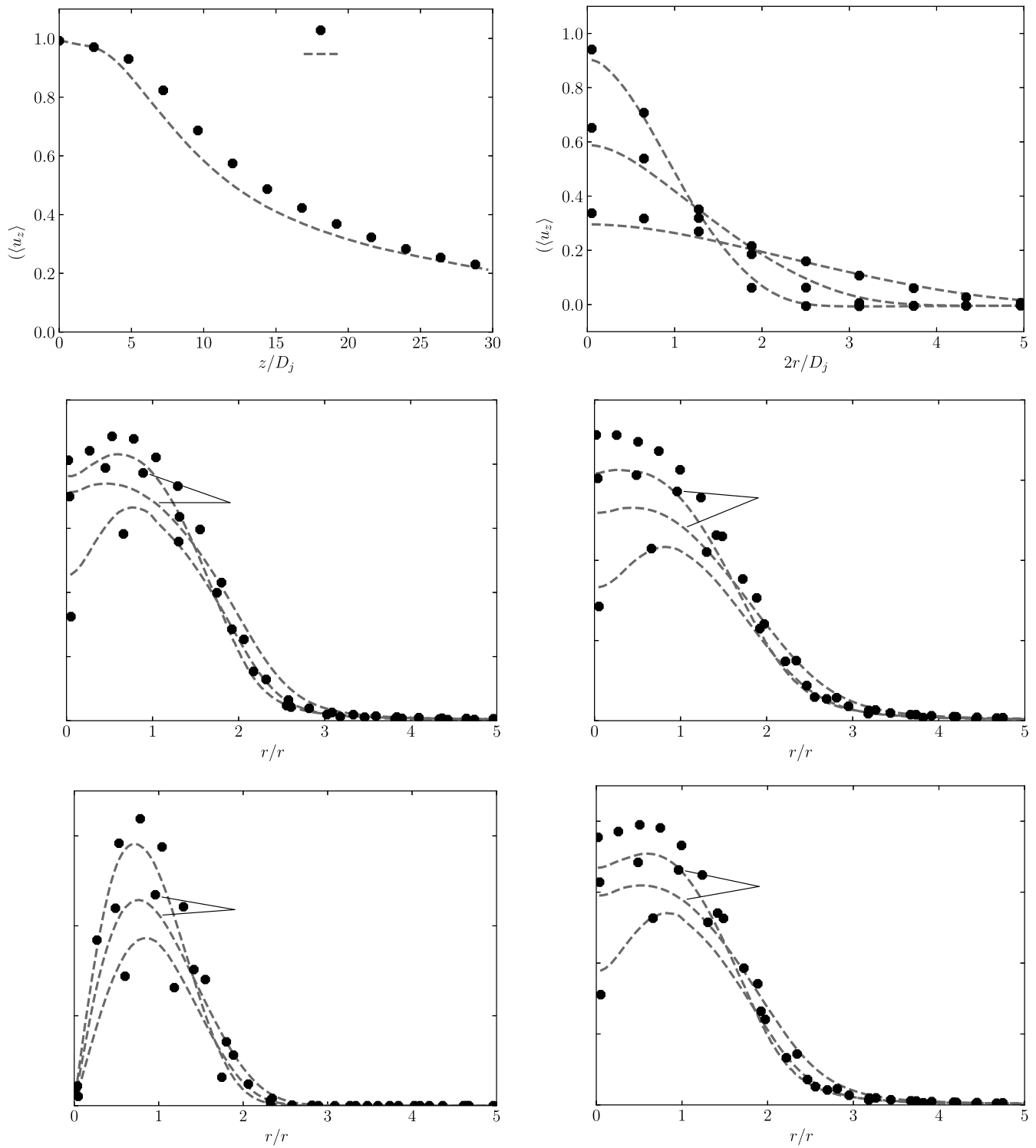

Figure 7: Comparison of WALE SGS model results against the numerical dataset. (a) Mean axial velocity along the jet axis. (b) Radial profiles of mean axial velocity at several axial positions. (c) Radial profiles of rms axial velocity at different axial positions. (d) Radial profiles of rms radial velocity at different axial positions. (e) Radial profiles of Reynolds shear-stress at different axial positions. (f) Radial profiles of turbulent kinetic energy at different axial positions. 

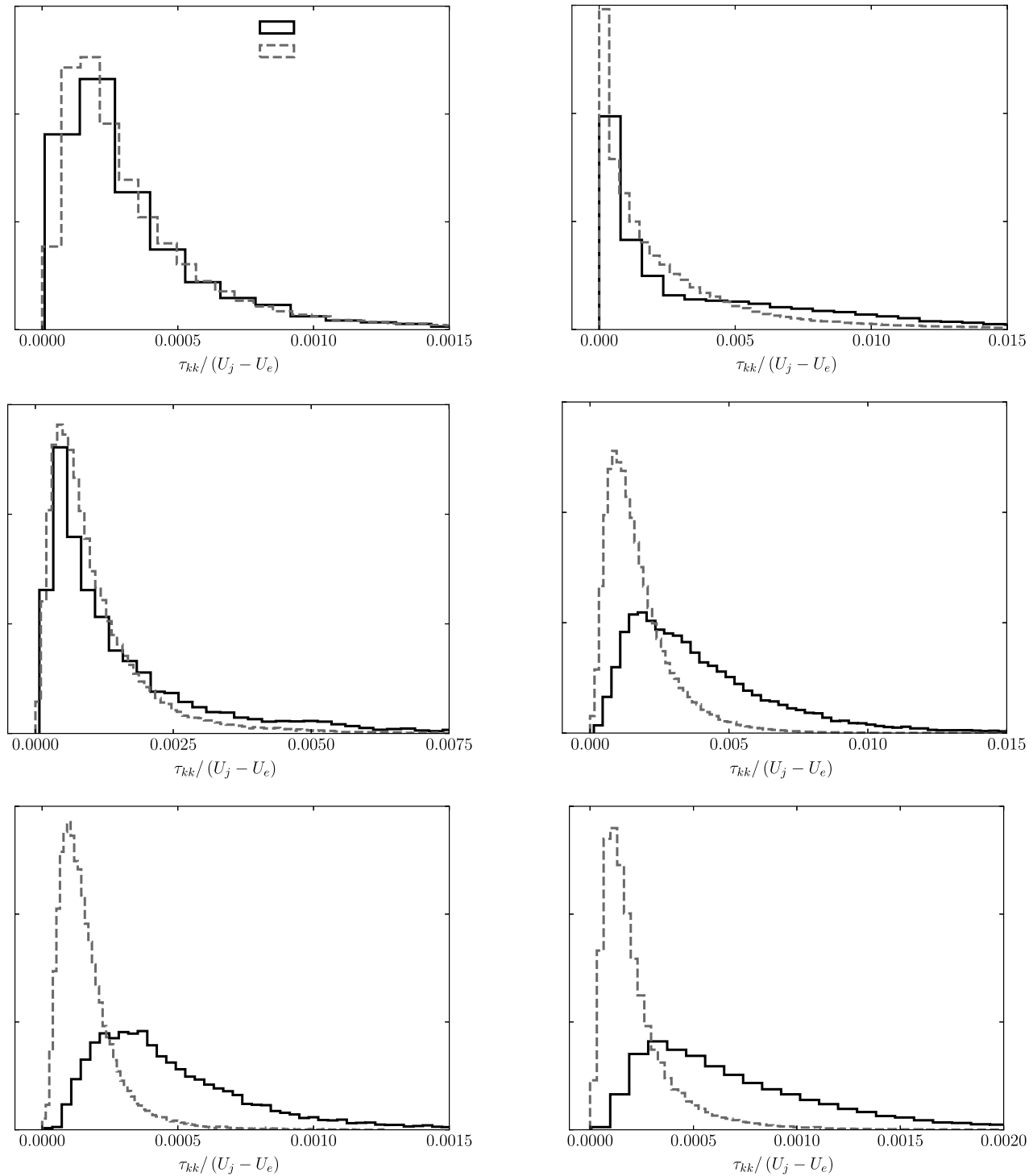

Figure 8: PDF of filtered and modeled $\tau_{k k}$ normalized by $\left(U_{j}-U_{e}\right)^{2}$ at different axial and radial positions. Rows: (top) $z / D_{j} \approx 1$, (center) $z / D_{j} \approx 5$, (bottom) $z / D_{j} \approx 20$. Columns: (left) $r / r_{1 / 2} \approx 0$, (right) $r / r_{1 / 2} \approx 1$. 

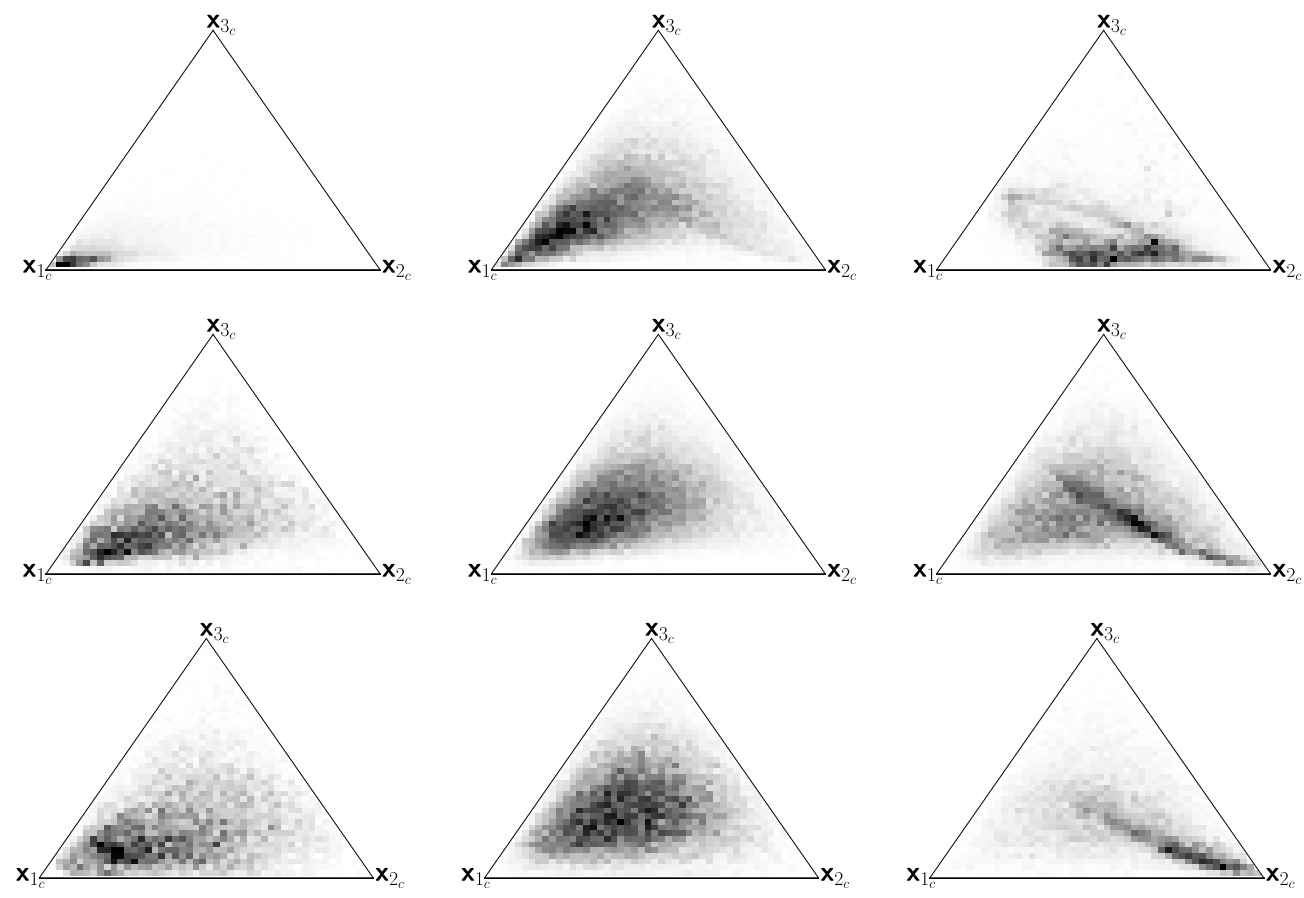

Figure 9: PDF of $\bar{\tau}_{i j}$ anisotropy represented on the barycentric map at different axial and radial positions. Rows: (top) $z / D_{j} \approx 1$, (center) $z / D_{j} \approx 5$, (bottom) $z / D_{j} \approx 20$. Columns: (left) $r / r_{1 / 2} \approx 0$, (center) $r / r_{1 / 2} \approx 1$, (right) $r / r_{1 / 2} \approx 2$. 

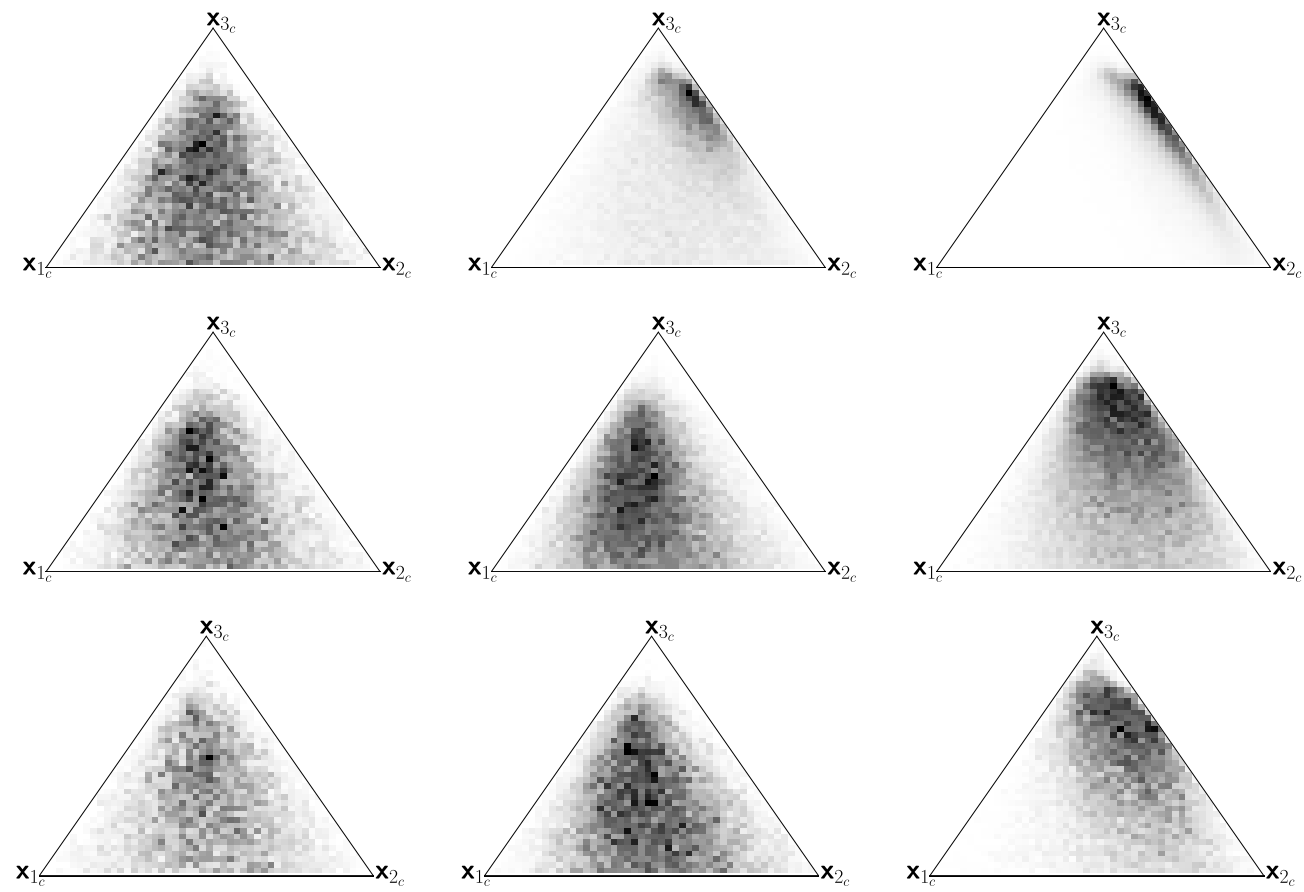

Figure 10: PDF of WALE $\tau_{i j}^{S G S}$ anisotropy represented on the barycentric map at different axial and radial positions. Rows: (top) $z / D_{j} \approx 1$, (center) $z / D_{j} \approx 5$, (bottom) $z / D_{j} \approx 20$. Columns: (left) $r / r_{1 / 2} \approx 0$, (center) $r / r_{1 / 2} \approx 1$, (right) $r / r_{1 / 2} \approx 2$.
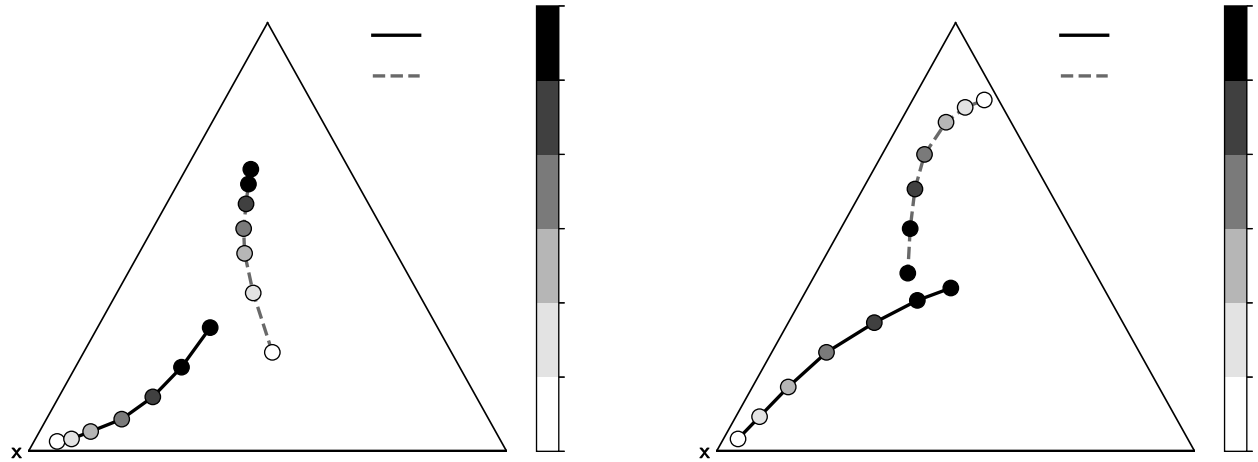

Figure 11: Trajectories on the barycentric map of mean $\tau_{i j}$ anisotropy in the axial direction at radial positions $r / r_{1 / 2} \approx 0$ (a) and $r / r_{1 / 2} \approx 1$ (b). 

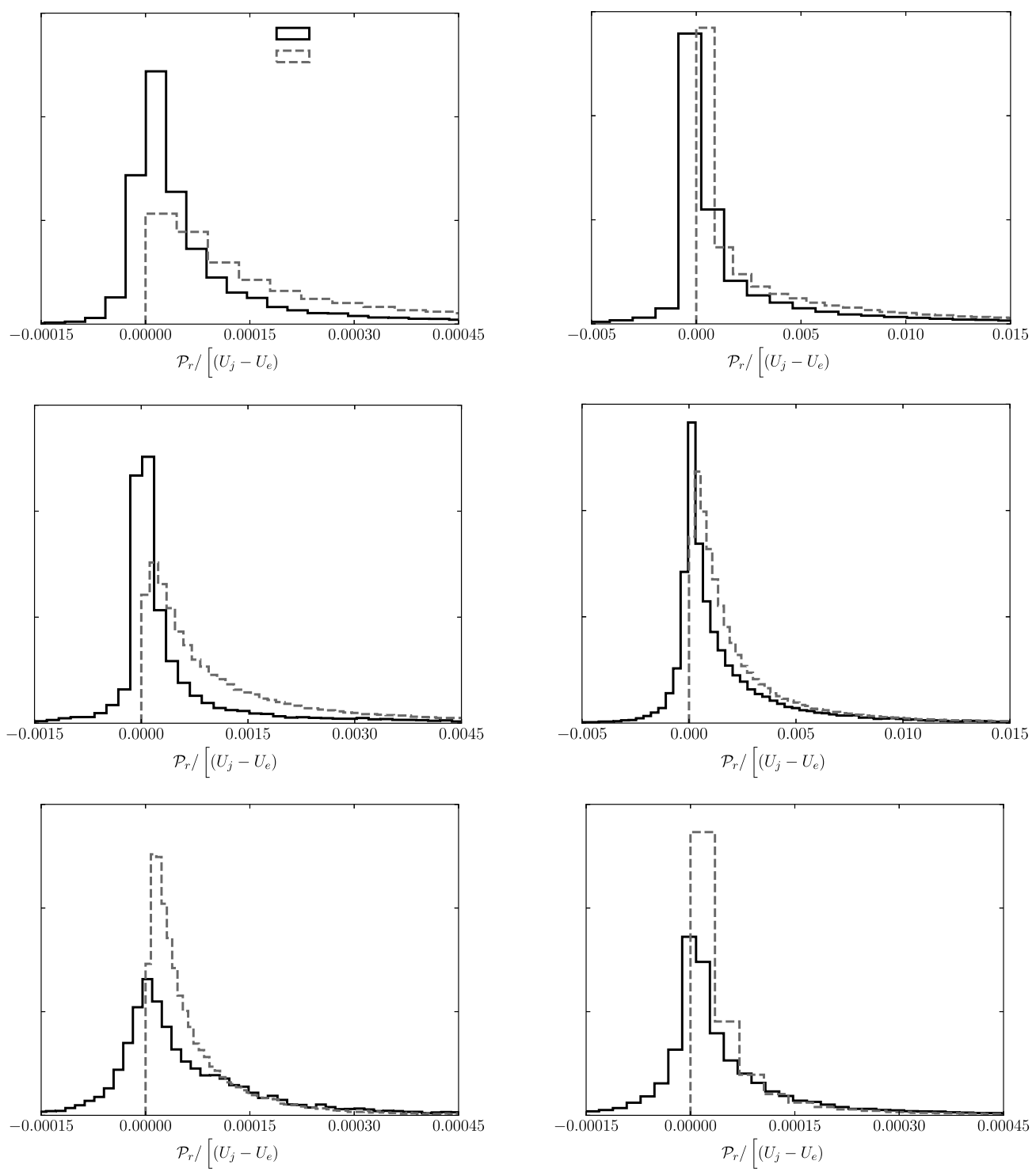

Figure 12: PDF of filtered and modeled $\mathcal{P}_{r}$ normalized by $\left(U_{j}-U_{e}\right)^{3} / D_{j}$ at different axial and radial positions. Rows: (top) $z / D_{j} \approx 1$, (center) $z / D_{j} \approx 5$, (bottom) $z / D_{j} \approx 20$. Columns: (left) $r / r_{1 / 2} \approx 0$, (right) $r / r_{1 / 2} \approx 1$. 

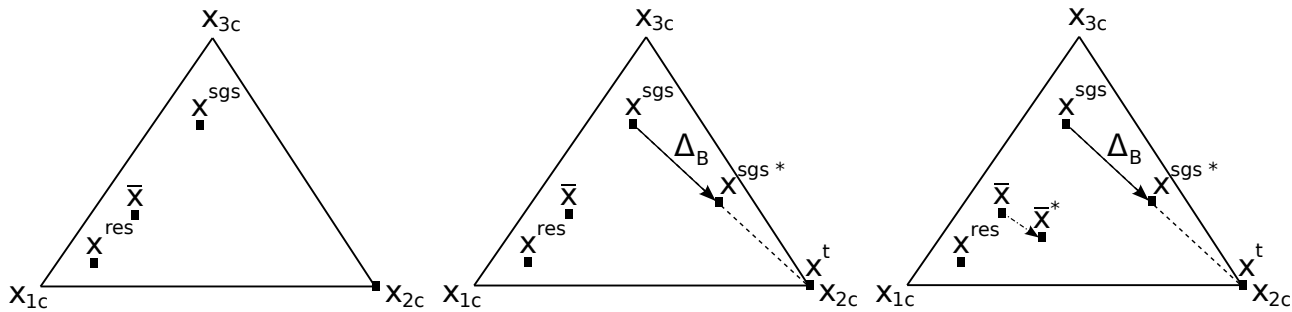

Figure 13: Sequential illustration of the eigenvalue perturbation procedure. The resolved, $\mathbf{x}^{\text {res }}$, and SGS base-model, $\mathbf{x}^{S G S}$, parts provide an initial location $\overline{\mathbf{x}}$ within the triangle (left). A perturbation of magnitude $\Delta_{B}$ toward $\mathbf{x}_{2 c}$ is applied to $\mathbf{x}^{S G S}$ (center). The new location of the SGS part, $\mathbf{x}^{S G S^{*}}$, indirectly modifies the coordinates of $\overline{\mathbf{x}}$, resulting in a perturbed state $\overline{\mathbf{x}}^{*}$ (right).
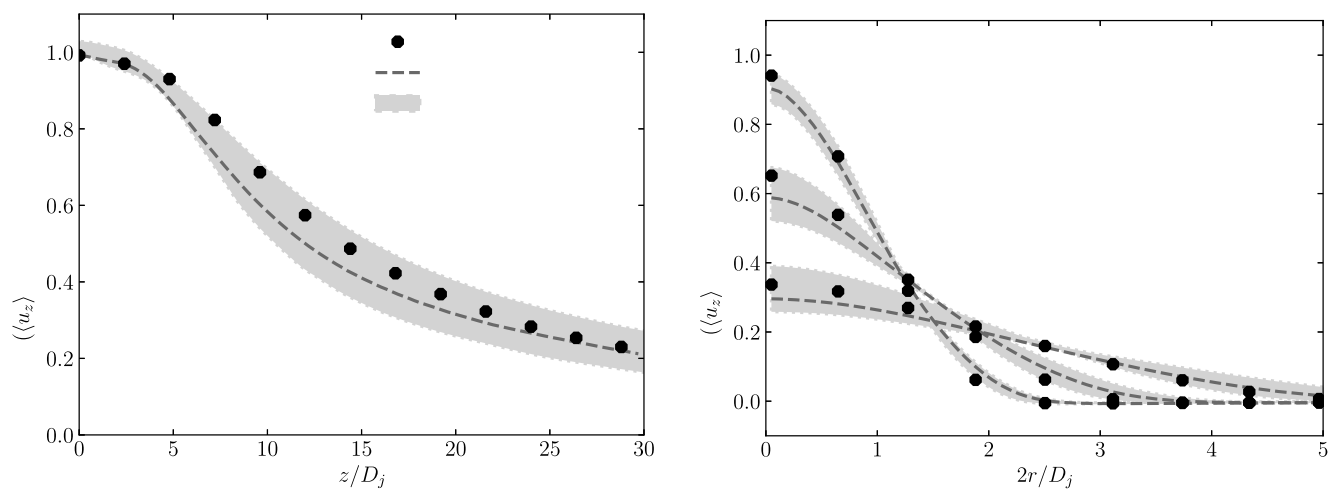

Figure 14: Comparison of WALE SGS uncertainty estimates against the numerical dataset. (a) Mean axial velocity along jet axis. (b) Radial profiles of mean axial velocity at several axial positions. 

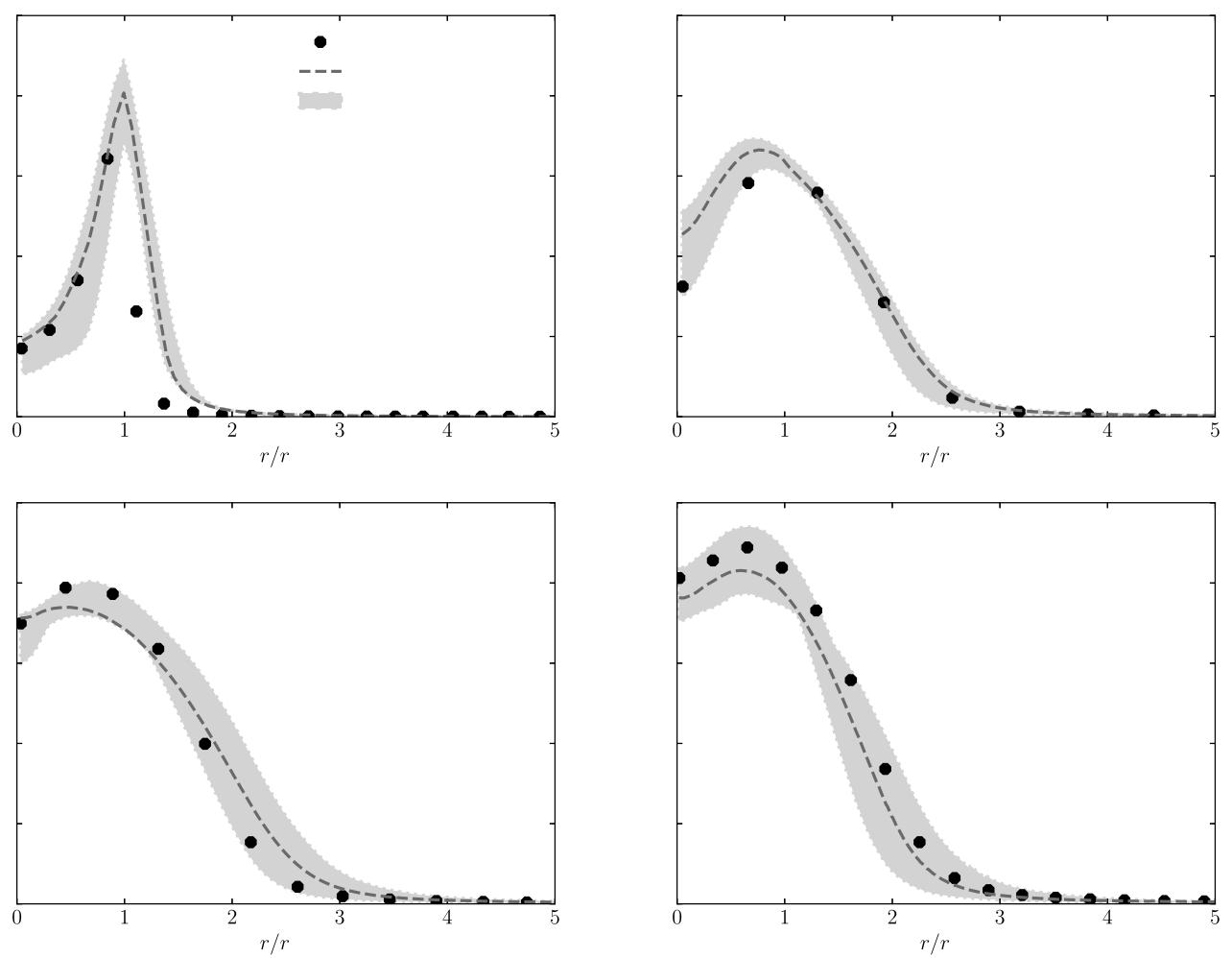

Figure 15: Comparison of WALE SGS uncertainty estimates against the numerical dataset. Radial profiles of rms axial velocity at axial positions: (a) $z / D_{j} \approx 1$, (b) $z / D_{j} \approx 5$, (c) $z / D_{j} \approx 10,(\mathrm{~d}) z / D_{j} \approx 20$. 

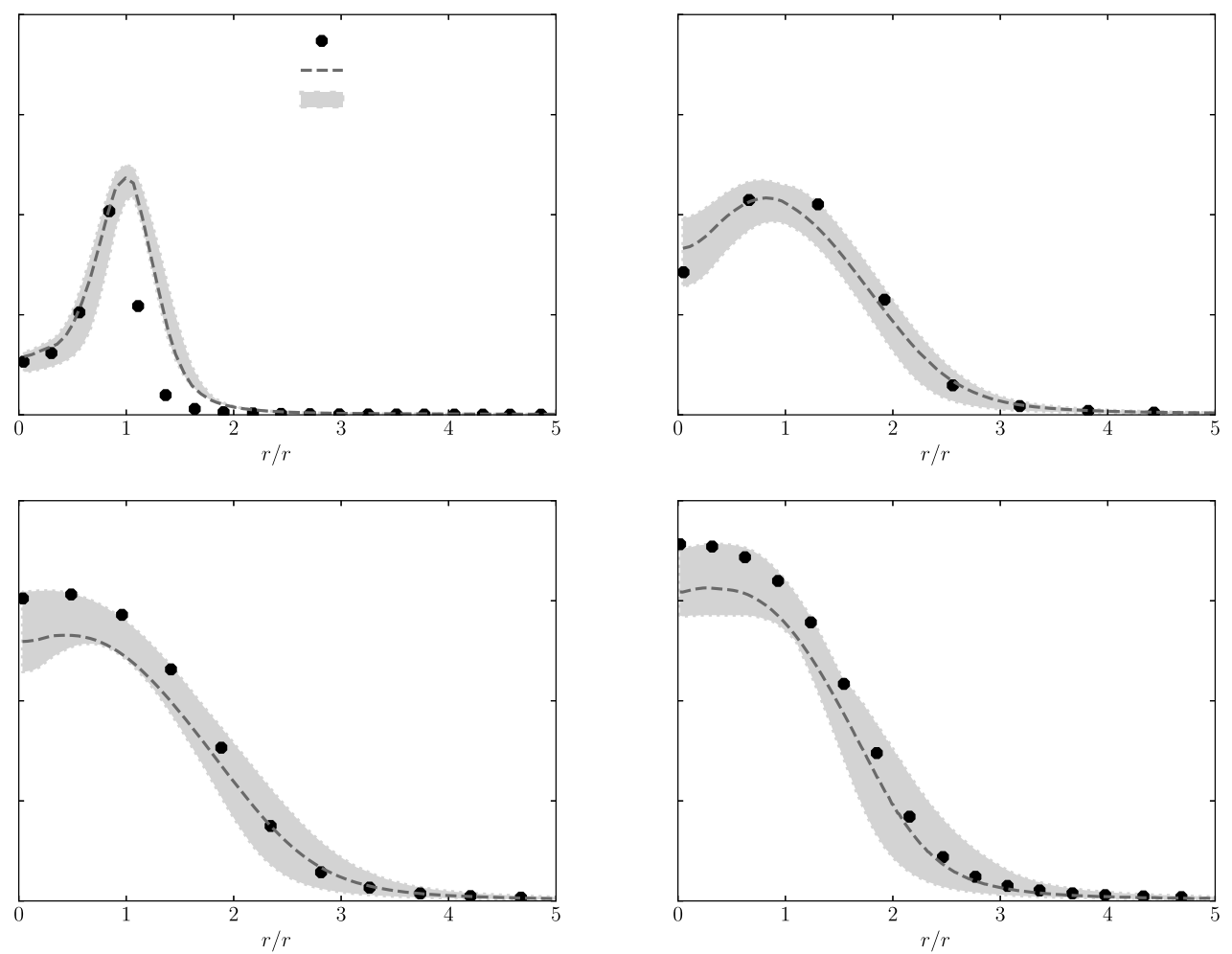

Figure 16: Comparison of WALE SGS uncertainty estimates against the numerical dataset. Radial profiles of rms radial velocity at axial positions: (a) $z / D_{j} \approx 1$, (b) $z / D_{j} \approx 5$, (c) $z / D_{j} \approx 10,(\mathrm{~d}) z / D_{j} \approx 20$. 

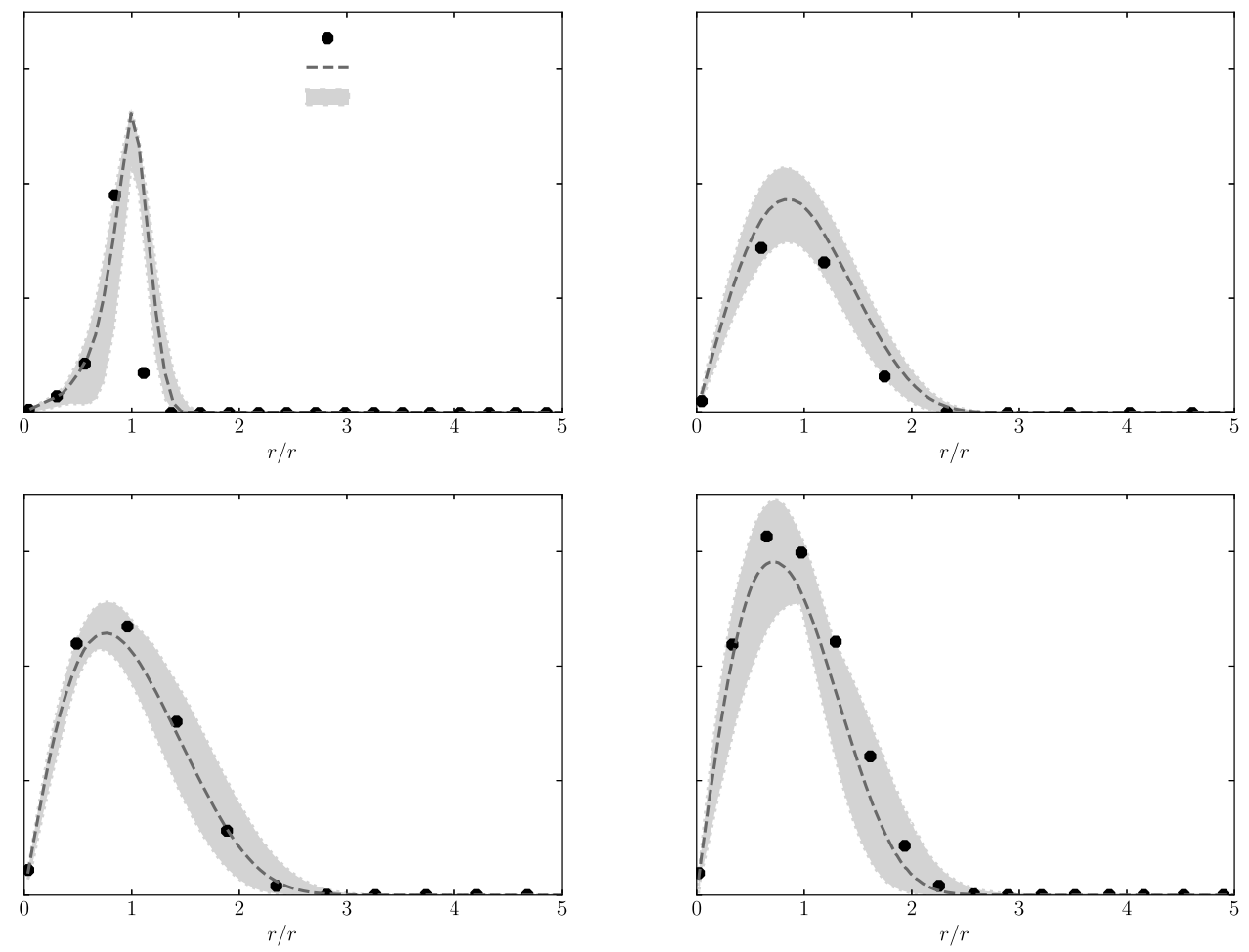

Figure 17: Comparison of WALE SGS uncertainty estimates against the numerical dataset. Radial profiles of Reynolds shear stress at axial positions: (a) $z / D_{j} \approx 1$, (b) $z / D_{j} \approx 5$, (c) $z / D_{j} \approx 10,(\mathrm{~d}) z / D_{j} \approx 20$. 

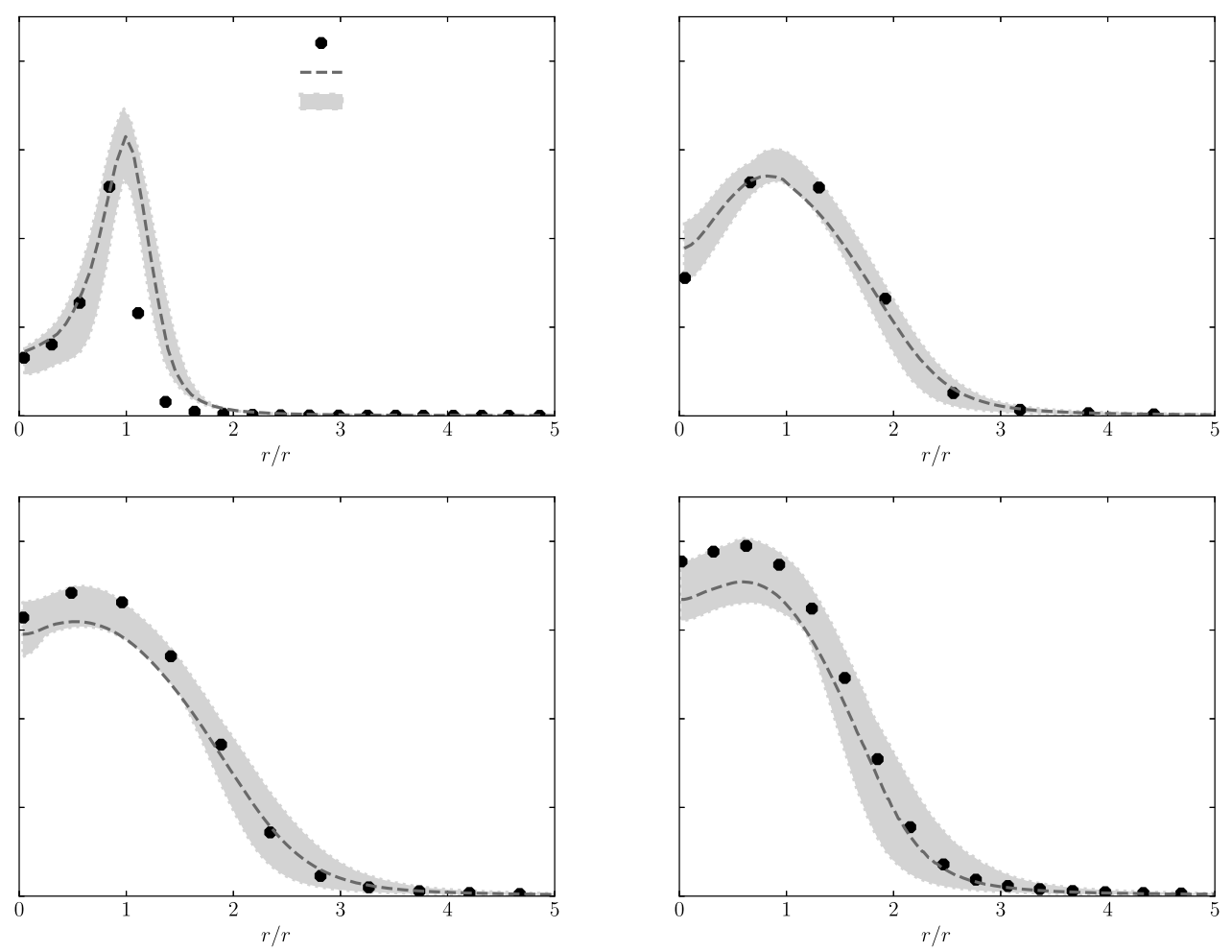

Figure 18: Comparison of WALE SGS uncertainty estimates against the numerical dataset. Radial profiles of turbulent kinetic energy at axial positions: (a) $z / D_{j} \approx 1$, (b) $z / D_{j} \approx 5$, (c) $z / D_{j} \approx 10$, (d) $z / D_{j} \approx 20$. 

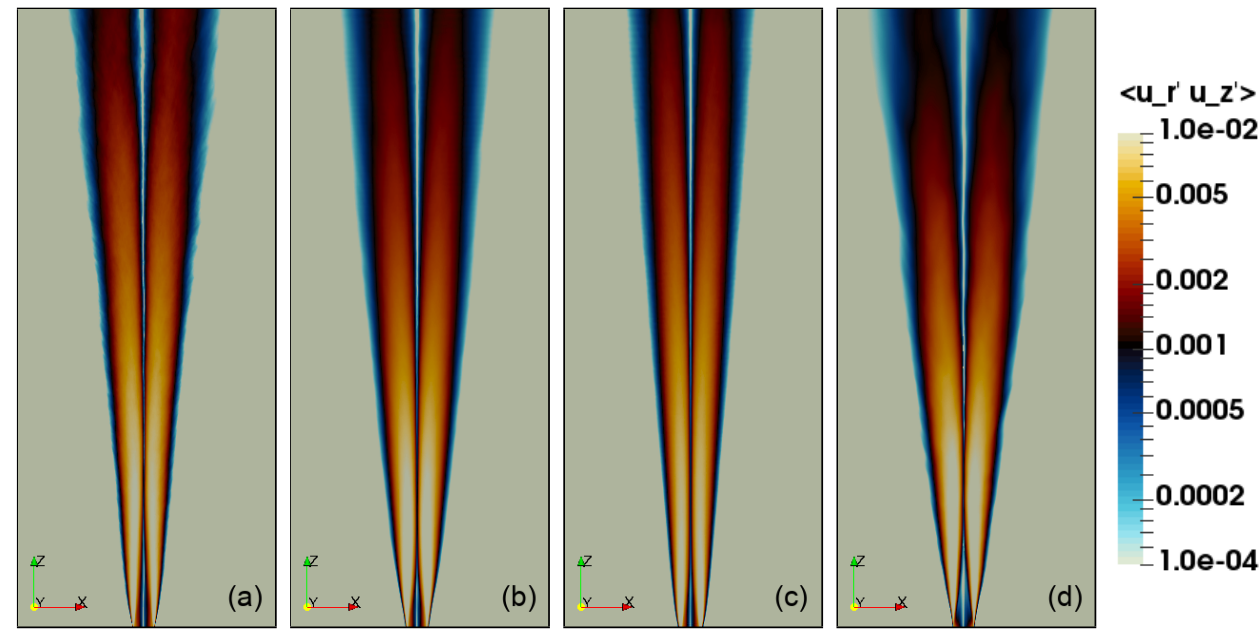

Figure 19: Normalized Reynolds shear-stress, $\left\langle u_{r}^{\prime} u_{z}^{\prime}\right\rangle /\left(U_{j}-U_{e}\right)^{2}$, visualized on the $x z$ plane. (a) Reference numerical dataset. (b) WALE SGS model. (c) WALE SGS model with $\Delta \tau_{k k}^{S G S} \leq 0$ magnitude perturbation. (d) WALE SGS model with anisotropy perturbation toward vertex $\mathbf{x}_{1 c}$.
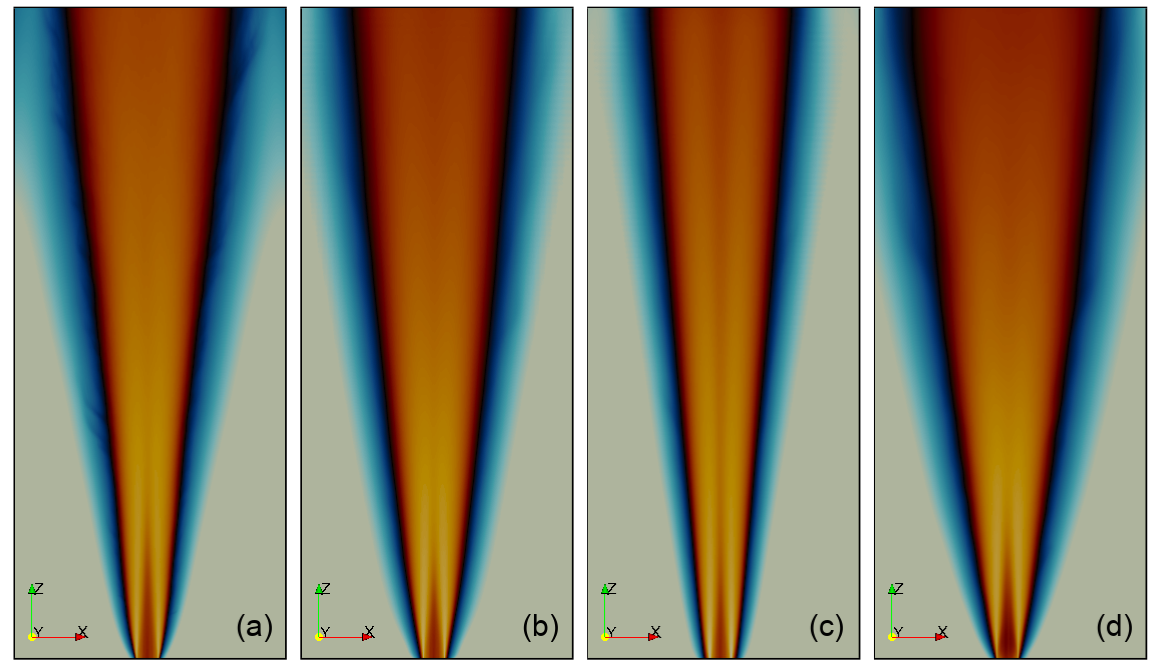

TKE (norm.)
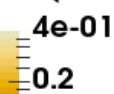

$=0.1$

0.05

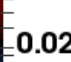

0.02

0.01

0.005

0.002

Ele-03

Figure 20: Normalized, resolved turbulent kinetic energy, $(2 k / 3)^{1 / 2} /\left(U_{j}-U_{e}\right)$, visualized on the $x z$-plane. (a) Reference numerical dataset. (b) WALE SGS model. (c) WALE SGS model with $\Delta \tau_{k k}^{S G S} \leq 0$ magnitude perturbation. (d) WALE SGS model with anisotropy perturbation toward vertex $\mathbf{x}_{1 c}$. 

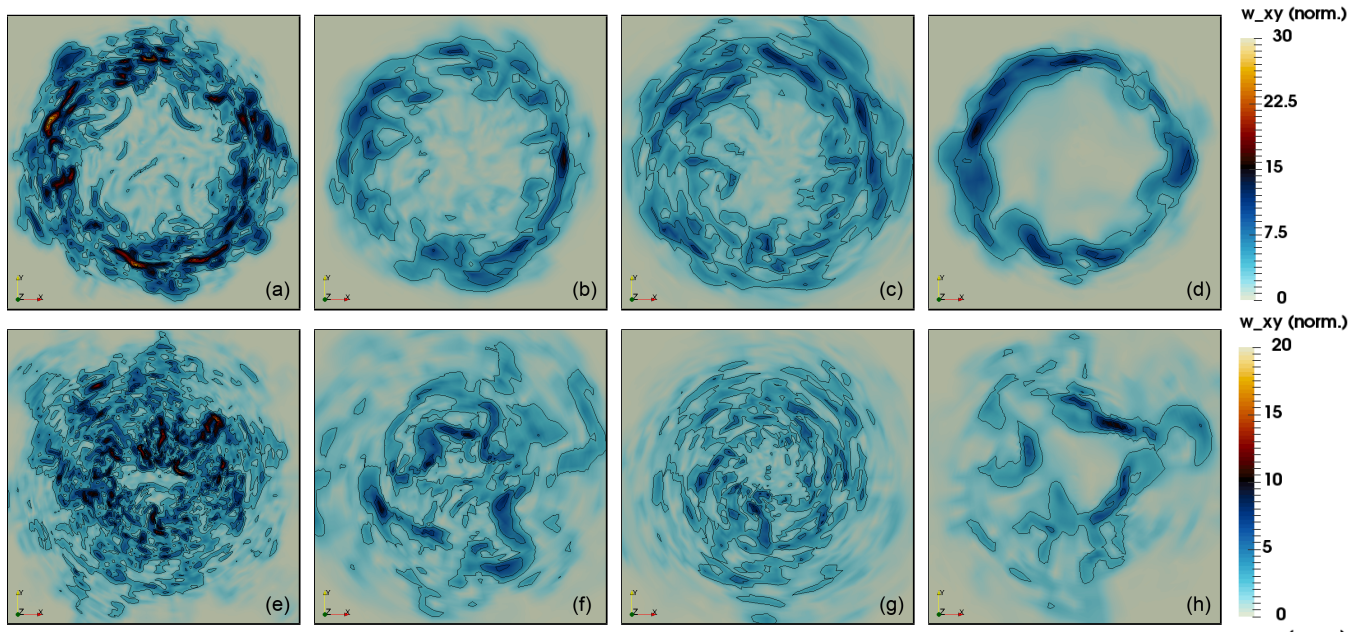

20
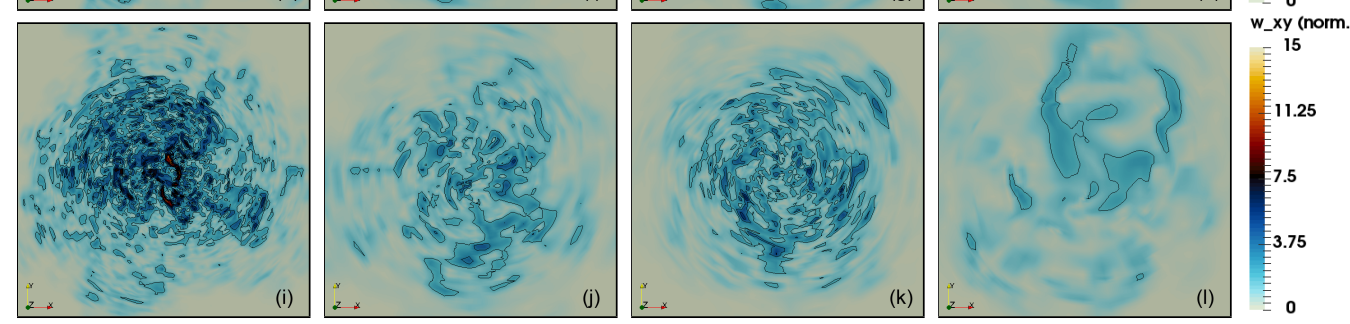

Figure 21: Instantaneous normalized, resolved $x y$-plane vorticity, $\left(\bar{\omega}_{x}^{2}+\bar{\omega}_{y}^{2}\right)^{1 / 2} /\left[\left(U_{j}-U_{e}\right) / D_{j}\right]$, visualized at different axial distances. Rows: (top) $z / D_{j}=1$, (center) $z / D_{j}=5$, (bottom) $z / D_{j}=10$. Columns: (left to right) reference numerical dataset, WALE SGS model, WALE SGS model with $\Delta \tau_{k k}^{S G S} \leq 0$ magnitude perturbation, WALE SGS model with anisotropy perturbation toward vertex $\mathbf{x}_{1 c}$. 

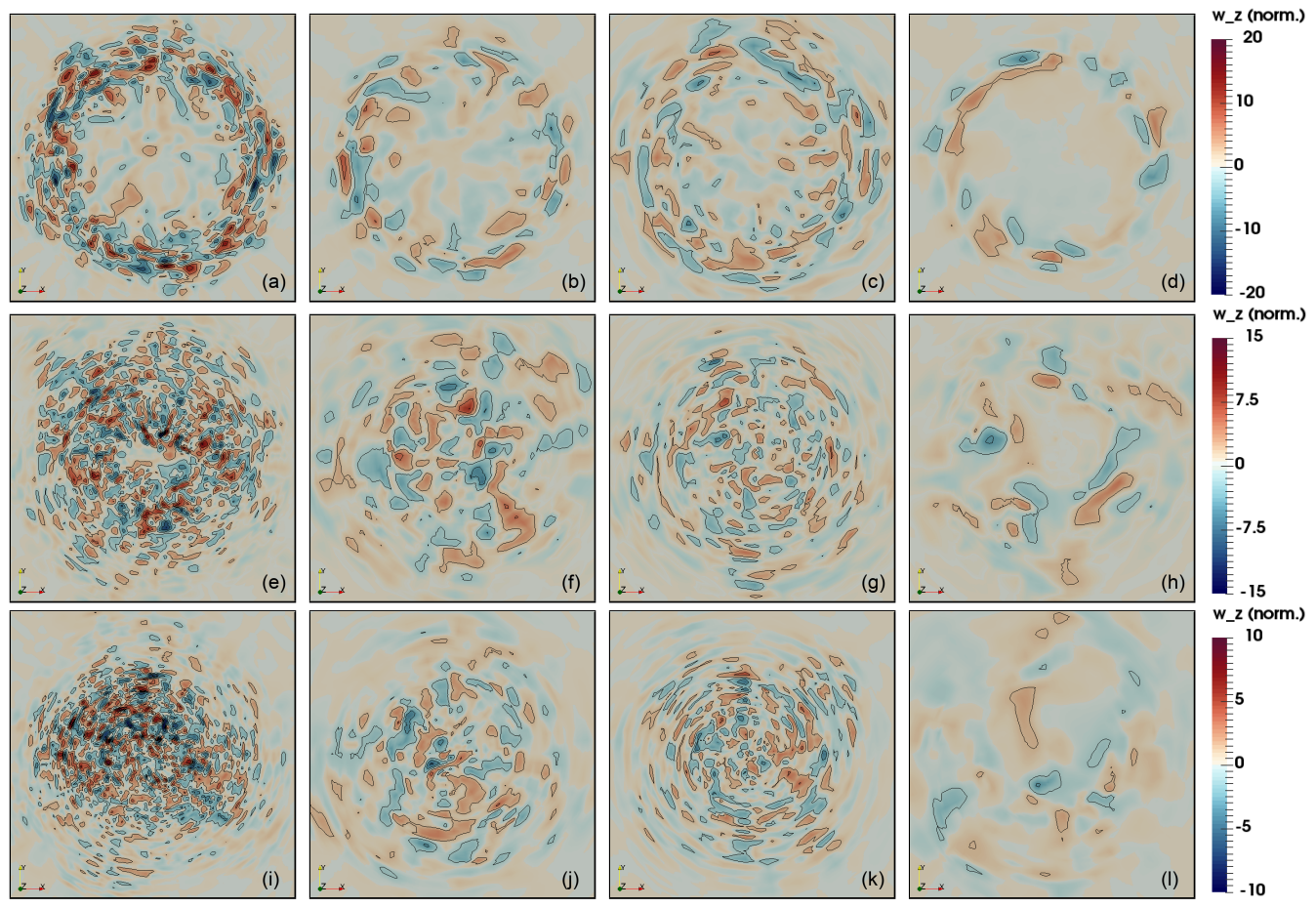

Figure 22: Instantaneous normalized, resolved $z$ vorticity, $\bar{\omega}_{z} /\left[\left(U_{j}-U_{e}\right) / D_{j}\right]$, visualized at different axial distances. Rows: (top) $z / D_{j}=1$, (center) $z / D_{j}=5$, (bottom) $z / D_{j}=$ 10. Columns: (left to right) reference numerical dataset, WALE SGS model, WALE SGS model with $\Delta \tau_{k k}^{S G S} \leq 0$ magnitude perturbation, WALE SGS model with anisotropy perturbation toward vertex $\mathbf{x}_{1 c}$. 

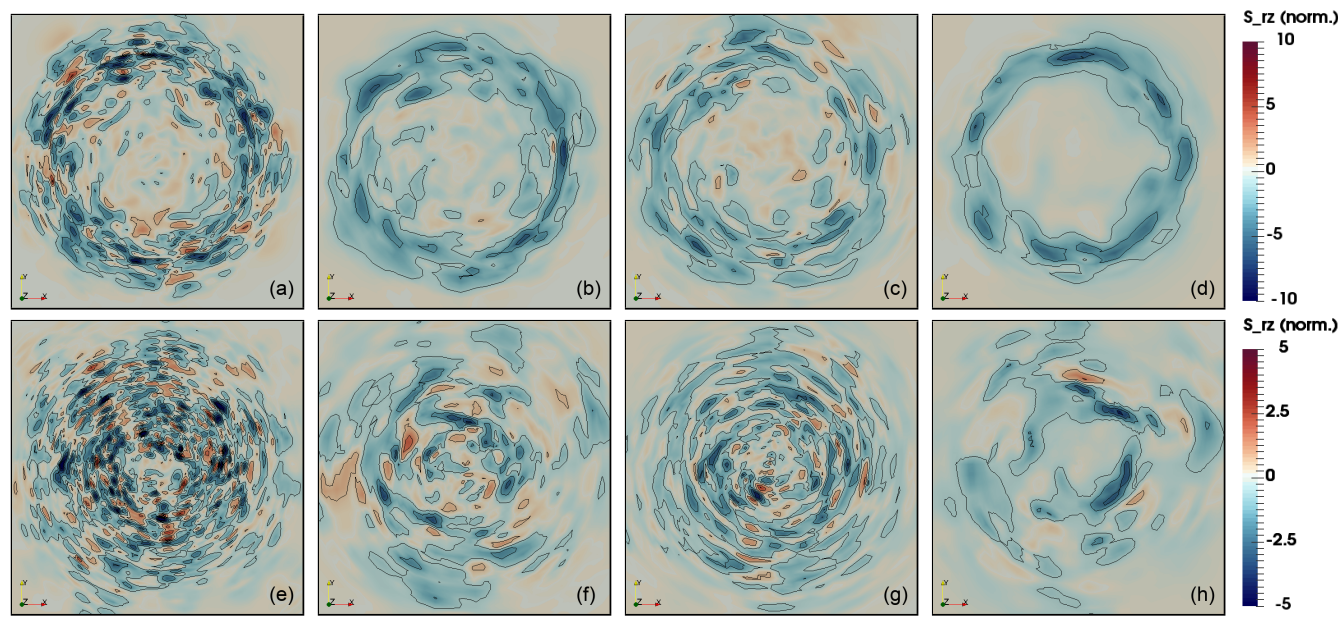

S_lz (norm)
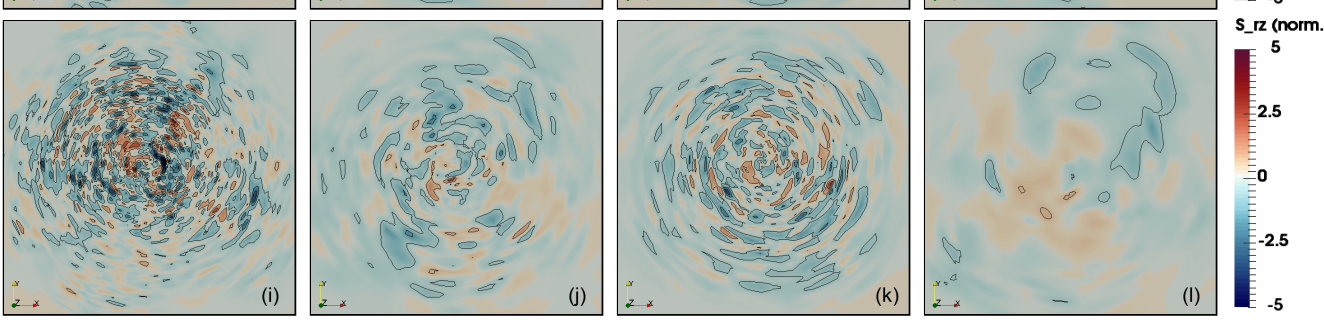

Figure 23: Instantaneous normalized, resolved $r z$ rate-of-strain, $\bar{S}_{r z} /\left[\left(U_{j}-U_{e}\right) / D_{j}\right]$, visualized at different axial distances. Rows: (top) $z / D_{j}=1$, (center) $z / D_{j}=5$, (bottom) $z / D_{j}=10$. Columns: (left to right) reference numerical dataset, WALE SGS model, WALE SGS model with $\Delta \tau_{k k}^{S G S} \leq 0$ magnitude perturbation, WALE SGS model with anisotropy perturbation toward vertex $\mathbf{x}_{1 c}$. 